\title{
On threshold solutions of the equivariant Chern-Simons-Schrödinger equation
}

\author{
Zexing Li and Baoping Liu
}

\begin{abstract}
We consider the self-dual Chern-Simons-Schrödinger model in two spatial dimensions. This problem is $L^{2}$-critical. Under the equivariant setting, global well-posedness and scattering were proved in Liu and Smith (2016) for a solution with initial charge below a certain threshold given by the ground state. In this work, we show that the only nonscattering solutions with threshold charge are exactly the ground state up to scaling, phase rotation and the pseudoconformal transformation. We also obtain a partial result for the non-self-dual system.
\end{abstract}

\section{Introduction}

\subsection{Covariant formulation}

The Chern-Simons-Schrödinger equation is a nonrelativistic quantum model describing the dynamics of a large number of charged particles in the plane interacting both directly and via a self-generated electromagnetic field. The model is a Lagrangian field theory on $\mathbb{R}^{1+2}$ associated to the action

$$
\begin{aligned}
L[A, \phi]= & \frac{1}{2} \int_{\mathbb{R}^{1+2}}\left[\operatorname{Im}\left(\bar{\phi} \boldsymbol{D}_{t} \phi\right)+\left|\boldsymbol{D}_{x} \phi\right|^{2}-\frac{g}{2}|\phi|^{4}\right] d x d t \\
& +\frac{1}{2} \int_{\mathbb{R}^{1+2}} A \wedge d A .
\end{aligned}
$$

Here, $\phi: \mathbb{R}^{1+2} \rightarrow \mathbb{C}$ is a scalar field describing the particle system, the potential $A:=$ $A_{0} d t+A_{1} d x_{1}+A_{2} d x_{2}$ is a real-valued 1 -form on $\mathbb{R}^{1+2}$, the associated covariant differentiation operators $\boldsymbol{D}_{\alpha}:=\partial_{\alpha}+i A_{\alpha}$ for $\alpha \in\{0,1,2\}$ are defined in terms of the potential $A$, and $g \in \mathbb{R}$ is a coupling constant. For indices we use $\alpha=0$ for the time variable and $\alpha=1,2$ for the spatial variables $x_{1}, x_{2}$. The Lagrangian is invariant with respect to the transformation

$$
\phi \mapsto e^{-i \theta} \phi, \quad A \mapsto A+d \theta
$$

for a compactly supported real-valued function $\theta(t, x)$.

2020 Mathematics Subject Classification. 35Q55.

Keywords. Chern-Simons-Schrödinger equation, self-duality, minimal blowup solutions. 
Computing the Euler-Lagrange equation, we obtain the Chern-Simons-Schrödinger equation (CSS)

$$
\left\{\begin{array}{l}
\boldsymbol{D}_{t} \phi=i \boldsymbol{D}_{l} \boldsymbol{D}_{l} \phi+i g|\phi|^{2} \phi \\
F_{01}=-\operatorname{Im}\left(\bar{\phi} \boldsymbol{D}_{2} \phi\right) \\
F_{01}=\operatorname{Im}\left(\bar{\phi} \boldsymbol{D}_{1} \phi\right) \\
F_{12}=-\frac{1}{2}|\phi|^{2}
\end{array}\right.
$$

where $F=d A$ is the curvature 2-form, namely $F_{\alpha \beta}=\partial_{\alpha} A_{\beta}-\partial_{\beta} A_{\alpha}$. System (CSS) is a basic model of Chern-Simons dynamics $([18,19,26])$. For further physical motivation to study (CSS), such as quantum Hall effects, high temperature superconductivity and the quantization of Heisenberg ferromagnets, see [12,27, 28, 46, 56].

We have conservation laws for charge and energy,

$$
\begin{aligned}
\operatorname{chg}[\phi] & :=\int_{\mathbb{R}^{2}}|\phi|^{2} d x, \\
E[\phi] & :=\int_{\mathbb{R}^{2}}\left(\frac{1}{2}\left|\boldsymbol{D}_{x} \phi\right|^{2}-\frac{g}{4}|\phi|^{4}\right) d x .
\end{aligned}
$$

The system is $L^{2}$-critical in the sense that it admits a scaling transformation leaving the charge of $\phi$ and the equation invariant:

$$
(\phi, A) \mapsto\left\{\begin{array}{l}
\tilde{\phi}(t, x):=\lambda \phi\left(\lambda^{2} t, \lambda x\right), \\
\widetilde{A_{0}}(t, x):=\lambda^{2} A_{0}\left(\lambda^{2} t, \lambda x\right), \\
\widetilde{A_{j}}(t, x):=\lambda A_{j}\left(\lambda^{2} t, \lambda x\right) .
\end{array}\right.
$$

The property of this system changes when $g$ varies. Via the Bogomol'nyi identity (2.2), the dividing point is the self-dual case $g=1$, where the energy functional enjoys a complete square structure (2.5). Generally speaking, self-duality refers to theories in which interactions have particular forms and special strengths such that the second-order equation of motion reduces to the first, which is simpler to analyze. This feature draws crucial physical importance to models like self-dual Yang-Mills theory, self-dual YangMills-Higgs theory and self-dual Chern-Simons theory ([14]).

In this paper we impose the Coulomb gauge and restrict to the equivariant setting. We first rewrite (CSS) in the polar coordinates of $\mathbb{R}^{2}$. Define

$$
\partial_{r}=\frac{x_{1}}{|x|} \partial_{1}+\frac{x_{2}}{|x|} \partial_{2}, \quad \partial_{\theta}=-x_{2} \partial_{1}+x_{1} \partial_{2} .
$$

Correspondingly, we define

$$
\begin{array}{ll}
A_{r}=\frac{x_{1}}{|x|} A_{1}+\frac{x_{2}}{|x|} A_{2}, & A_{\theta}=-x_{2} A_{1}+x_{1} A_{2}, \\
\boldsymbol{D}_{r}=\frac{x_{1}}{|x|} \boldsymbol{D}_{1}+\frac{x_{2}}{|x|} \boldsymbol{D}_{2}, & \boldsymbol{D}_{\theta}=-x_{2} \boldsymbol{D}_{1}+x_{1} \boldsymbol{D}_{2} .
\end{array}
$$


We can formulate (CSS) equivalently as

$$
\left\{\begin{array}{l}
\boldsymbol{D}_{t} \phi=i\left(\boldsymbol{D}_{r}^{2}+\frac{1}{r} \boldsymbol{D}_{r}+\frac{1}{r^{2}} \boldsymbol{D}_{\theta}^{2}\right) \phi+i g|\phi|^{2} \phi, \\
\partial_{t} A_{r}-\partial_{r} A_{0}=-\frac{1}{r} \operatorname{Im}\left(\bar{\phi} \boldsymbol{D}_{\theta} \phi\right), \\
\partial_{t} A_{\theta}-\partial_{\theta} A_{0}=r \operatorname{Im}\left(\bar{\phi} \boldsymbol{D}_{r} \phi\right), \\
\partial_{r} A_{\theta}-\partial_{\theta} A_{r}=-\frac{1}{2} r|\phi|^{2},
\end{array}\right.
$$

with energy taking the form

$$
E[\phi]=\int_{\mathbb{R}^{2}}\left(\frac{1}{2}\left|\boldsymbol{D}_{r} \phi\right|^{2}+\frac{1}{2 r^{2}}\left|\boldsymbol{D}_{\theta} \phi\right|^{2}-\frac{g}{4}|\phi|^{4}\right) d x .
$$

Now we introduce the $m$-equivariant $(m \in \mathbb{Z})$ ansatz: ${ }^{1}$

$$
\begin{array}{ll}
\phi(t, x)=e^{i m \theta} u(t, r), & A_{1}(t, x)=-\frac{x_{2}}{r} v(t, r), \\
A_{2}(t, x)=\frac{x_{1}}{r} v(t, r), & A_{0}(t, x)=w(t, r) .
\end{array}
$$

The equivariant solutions of the Chern-Simons-Schrödinger system are called vortex solutions and appear in various related physical contexts (for instance [11, 29, 51]). In addition, as a reasonable and effective simplification, equivariant reduction is also applied commonly to other geometric equations, for example Chern-Simons-Higgs ([9]), the wave map ([10,52]) and the Schrödinger map ([2,8]). Also note that our formulation (1.7) implicitly indicates that we have chosen the Coulomb gauge condition ${ }^{2}$

$$
\nabla \cdot A_{x}=0
$$

Then (1.7) and (1.5) imply that

$$
A_{r}=0, \quad \partial_{r} A_{0}=\frac{1}{r}\left(m+A_{\theta}\right)|\phi|^{2}, \quad \partial_{r} A_{\theta}=-\frac{1}{2} r|\phi|^{2} .
$$

We make the natural boundary condition that $A_{0}$ decays to zero at spatial infinity (see [4] for further discussion). Hence, we obtain explicit formulas for $A_{\theta}$ and $A_{0}$,

$$
\begin{aligned}
& A_{\theta}[u](t, r)=-\frac{1}{2} \int_{0}^{r}|u(t, s)|^{2} s d s, \\
& A_{0}[u](t, r)=-\int_{r}^{\infty}\left(m+A_{\theta}[u](t, s)\right)|u(t, s)|^{2} \frac{d s}{s} .
\end{aligned}
$$

\footnotetext{
${ }^{1}$ We will often denote the radial part of $\phi$ by $u$ and will not distinguish unless necessary. We also remark that the equivariant assumption involves the radial case as $m=0$.

${ }^{2}$ Conversely, this ansatz can be derived from the Coulomb gauge condition plus the equivariant assumption merely on $\phi$. See [35] for details.
} 
Now we can rewrite the Chern-Simons-Schrödinger equation under the $m$-equivariant assumption as the $\phi$-evolution

$$
\left(i \partial_{t}+\Delta\right) \phi=\frac{2 m}{r^{2}} A_{\theta} \phi+A_{0} \phi+\frac{1}{r^{2}} A_{\theta}^{2} \phi-g|\phi|^{2} \phi,
$$

or the $u$-evolution

$$
\left(i \partial_{t}+\Delta_{m}\right) u=\frac{2 m}{r^{2}} A_{\theta} u+A_{0} u+\frac{1}{r^{2}} A_{\theta}^{2} u-g|u|^{2} u,
$$

where

$$
\Delta_{m}:=\partial_{r}^{2}+\frac{1}{r} \partial_{r}-\frac{m^{2}}{r^{2}}
$$

is the Laplacian for $m$-equivariant functions in $\mathbb{R}^{2}$. Also, we denote the nonlinear part by

$$
F(\phi):=\frac{2 m}{r^{2}} A_{\theta} \phi+A_{0} \phi+\frac{1}{r^{2}} A_{\theta}^{2} \phi-g|\phi|^{2} \phi,
$$

which is still an $m$-equivariant function. In this article we will focus on (eCSS). We will further restrict to the physically relevant cases $m \geq 0$ (see [14]).

\subsection{Known results and the threshold problem}

The Chern-Simons-Schrödinger system (CSS) has drawn much attention since the 1990s. Under the Coulomb gauge, local well-posedness was first established with initial data in $H^{2}$ by Bergé-de Bouard-Saut ([4]). For $H^{1}$ initial data with small charge, they also obtained global existence (but without uniqueness). Huh ([23]) showed that (CSS) has a unique local-in-time solution for $H^{1}$ data, without continuous dependence. Lim ([43]) obtained $H^{1}$ local well-posedness with weak Lipschitz dependence for small $L^{2}$ data. Using the heat gauge, Liu-Smith-Tataru ([45]) established local well-posedness and strong Lipschitz dependence in $H^{\varepsilon}, \varepsilon>0$ for small $H^{\varepsilon}$ data. In addition, Oh-Pusateri ([50]) proved global existence and scattering for solutions with small data in weighted Sobolev spaces, by revealing a cubic null structure under the Coulomb gauge. So far, well-posedness for (CSS) at the critical regularity in any gauge remains an interesting open problem.

Under the equivalence setting, Liu-Smith ([44]) demonstrated that the local wellposedness of (eCSS) with $L^{2}$ data can be proved via mere Strichartz estimates. Moreover, a threshold result is obtained in [44].

To explain the result, we first note that for $g \geq 1$, (eCSS) admits soliton solutions. Consider the elliptic equation

$$
\Delta_{m} u-\alpha u-\frac{2 m}{r^{2}} A_{\theta}[u] u-A_{0}[u] u-\frac{1}{r^{2}} A_{\theta}[u]^{2} u+g|u|^{2} u=0
$$


with $\alpha \geq 0$. When $g=1$, Byeon-Huh-Seok ([5,6]) showed that (1.15) admits a unique positive $^{3}$ radial solution with $\alpha=0$,

$$
Q^{(m)}(r):=\sqrt{8}(m+1) \frac{r^{m}}{1+r^{2(m+1)}}
$$

under the boundary condition $A_{0} \rightarrow 0$ as $|x| \rightarrow+\infty$. We simplify the notation by writing $Q=Q^{(m)}$ when $m$ is fixed. This generates the static solution

$$
\phi^{(m)}(x):=e^{i m \theta} Q(r)
$$

to self-dual (eCSS). ${ }^{4}$

In [5, 6], the authors also proved the nonexistence of a solution for (1.15) when $g \in$ $(0,1)$ and the existence of a positive radial solution for (1.15) with $\alpha \geq 0$ as $g>1$, which we denote as $Q^{(m, g, \alpha)}{ }^{5}$ By writing $\phi^{(m, g, \alpha)}(x):=Q^{(m, g, \alpha)}(r) e^{i m \theta}$, we obtain either a static solution $(\alpha=0)$ or a stationary wave $(\alpha>0)$ to (eCSS) for $g>1$, which is of the form $\psi^{(m, g, \alpha)}(t, x):=\phi^{(m, g, \alpha)}(x) e^{i \alpha t}$ for some $\alpha \geq 0$. It is also conjectured in [5] that (eCSS) only admits a stationary wave when $g>1$.

In fact, these soliton solutions are the minimal-charge obstructions to global wellposedness and scattering as explained in the following threshold theorem. Let us first define the equivariant Sobolev space as

$$
H_{m}^{s}:=\left\{f \in H^{s}: \exists u=u(r) \text { s.t. } f(x)=e^{i m \theta} u(r)\right\}, \quad L_{m}^{2}:=H_{m}^{0} .
$$

The homogeneous Sobolev space $\dot{H}_{m}^{s}$ is also defined in this way. It is easy to see that $\|f\|_{\dot{H}_{m}^{1}}^{2}=\left\|\partial_{r} f\right\|_{L^{2}}^{2}+\left\|\frac{m}{r} f\right\|_{L^{2}}^{2}$.

Theorem 1.1 (Threshold result [44]). Let $m \in \mathbb{N}:=\{n \in \mathbb{Z}: n \geq 0\}$.

(1) Let $g<1$. Then for any initial data $\phi_{0} \in L_{m}^{2}$, the solution $\phi$ of (eCSS) is globally well posed and scatters both forward and backward in time.

(2) Let $g=1$. Then for any initial data $\phi_{0} \in L_{m}^{2}$ with $\operatorname{chg}\left(\phi_{0}\right)<\operatorname{chg}\left(Q^{(m)}\right)=$ $8 \pi(m+1)$, the solution $\phi$ of (eCSS) is globally well posed and scatters both forward and backward in time.

(3) Let $g>1$. Then there exists a constant $c_{m, g}>0$ such that for any initial data $\phi_{0} \in L_{m}^{2}$ with $\operatorname{chg}\left(\phi_{0}\right)<c_{m, g}$, the solution $\phi$ of (eCSS) is globally well posed and scatters both forward and backward in time. Moreover, the minimum charge of a nontrivial standing wave solution $\psi^{(m, g)}$ in the class $L_{t}^{\infty} L_{m}^{2}$ is equal to $c_{m, g}$.

\footnotetext{
${ }^{3}$ In fact, when $m>0$ we only have $Q>0$ on $\mathbb{R}^{2} \backslash\{0\}$. Indeed, the zero at the origin exists for any $m$ equivariant function $f$ to be continuous. So in the following text, when we say an $m$-equivariant $(m>0)$ function is positive, we will always mean it is positive in $\mathbb{R}^{2} \backslash\{0\}$.

${ }^{4}$ In the self-dual case, with the help of (2.5) one can show that zero energy solutions are gauge equivalent to static solutions of (CSS), even without the equivariant assumption. A rigorous proof can be found in $[24,35]$.
}

${ }^{5}$ Since $\alpha$ depends on $m$ and $g$, we also use the notation $Q^{(m, g)}:=Q^{(m, g, \alpha(m, g))}$ and similarly for $\phi, \psi$. 
Remark 1.2. By scattering forward/backward in time, we mean there exists $\phi_{ \pm} \in L^{2}$ such that

$$
\lim _{t \rightarrow \pm \infty}\left\|\phi(t)-e^{i t \Delta} \phi_{ \pm}\right\|_{L^{2}}=0 .
$$

In the proof of Theorem 1.1, the $L_{t, x}^{4}$ norm plays the role of a scattering norm in the following sense. Let $\phi: I \times \mathbb{R}^{2} \rightarrow \mathbb{C}$ be a solution to (eCSS), where $I$ is the maximal lifespan; if $\sup I=+\infty$ and $\|\phi\|_{L_{t, x}^{4}\left([0,+\infty) \times \mathbb{R}^{2}\right)}<\infty$, then the solution scatters forward in time.

For this reason, we say a solution $\phi$, with maximal lifespan $I$, blows up forward or backward in time if $\|\phi\|_{L_{t, x}^{4}\left(I_{ \pm} \times \mathbb{R}^{2}\right)}=\infty$, with $I_{+}=[0$, sup $I)$ and $I_{-}=(\inf I, 0]$. In particular, it contains two scenarios: to blow up at infinite time or at finite time.

Note that (eCSS) admits pseudoconformal symmetry. The pseudoconformal transformation $^{6}$

$$
\mathrm{PC}_{T}: \psi(t, x) \mapsto \frac{1}{T-t} e^{-i \frac{|x|^{2}}{4(T-t)}} \psi\left(\frac{t}{T(T-t)}, \frac{x}{T-t}\right) \quad \forall t<T
$$

keeps equation (eCSS) invariant and conserves the solution's charge. By applying it to the solitons $\psi^{(m, g)}(g \geq 1)$ as above, we get $\mathrm{PC}_{T}\left[\psi^{(m, g)}\right]$, another $m$-equivariant solution for (eCSS) with the threshold charge. It blows up at a finite time $t=T$, while in contrast, the soliton $\psi^{(m, g)}$ blows up at infinite time.

It is a natural question to study solutions above or at the threshold charge. Recently, Kim-Kwon ([34,35]) studied finite-time blowup solutions for the self-dual (CSS) under the equivariant setting $(m \geq 1)$. They constructed a pseudoconformal blowup solution with given asymptotic profile and studied its instability mechanism. Furthermore, they constructed a codimension 1 manifold yielding pseudoconformal blowup solutions. KimKwon-Oh ([36]) considered the radial case and constructed a data set that leads to blowup solutions whose blowup rate differs from the pseudoconformal rate by a power of logarithm.

On the other hand, our work focuses on the special role $\mathrm{PC}_{T}\left[\psi^{(m, g)}\right]$ plays. We present a characterization for $H_{m}^{1}$ solutions with exact threshold charge.

\subsection{Main result}

Our main result is that, in the self-dual case $g=1$, any blowup $H_{m}^{1}$ solution must be (1.17) up to symmetries.

Theorem 1.3 (Characterization of the threshold solution in the self-dual case). For $m \geq 1$, $g=1$ and initial data $\phi_{0} \in H_{m}^{1}\left(\mathbb{R}^{2}\right),\left\|\phi_{0}\right\|_{L^{2}}=\left\|Q^{(m)}\right\|_{L^{2}}$, one of the following three scenarios happens:

(1) $u$ equals the pseudoconformal transformation of the ground state $Q^{(m)}$ up to phase rotation and scaling.

${ }^{6}$ The pseudoconformal invariance actually holds for the general (CSS) ([22]). 
(2) $u$ equals the ground state $Q^{(m)}$ up to phase rotation and scaling.

(3) $u$ scatters both forward and backward in time.

And for $m=0, g=1$ and initial data $\phi_{0} \in H_{\text {rad }}^{1}\left(\mathbb{R}^{2}\right),\left\|\phi_{0}\right\|_{L^{2}}=\left\|Q^{0}\right\|_{L^{2}}$, only cases (2), (3) with $m=0$ can happen. In particular, the solution must exist globally.

Noticing that a nonscattering solution blows up either at infinite time or at finite time, this result comes down to the following two theorems.

Theorem 1.4 (Rigidity of blowup in finite time in the self-dual case). For $m \in \mathbb{N}, g=1$ and initial data $\phi_{0} \in H_{m}^{1}\left(\mathbb{R}^{2}\right),\left\|\phi_{0}\right\|_{L^{2}}=\left\|Q^{(m)}\right\|_{L^{2}}$, if the solution of (eCSS) $\phi$ blows up at $T>0$, i.e. $\|\phi\|_{L_{t, x}^{4}\left([0, T) \times \mathbb{R}^{2}\right)}=\infty$, then there exists $\gamma \in[0,2 \pi), \lambda \in \mathbb{R}_{+}$such that

$$
\phi(t, x)=e^{i \gamma} \mathrm{PC}_{T}\left[\lambda \phi^{(m)}(\lambda \cdot)\right](t x) \quad \forall t<T .
$$

Remark 1.5. It is easy to see from (1.16) and (1.18) that $Q^{(m)} \in H_{m}^{1}$ for all $m \geq 0$ and $\mathrm{PC}_{T}\left[Q^{(m)}\right] \in H_{m}^{1}$ only for $m \geq 1$. So for $m=0$, Theorem 1.4 indicates that any threshold solution generated by $\phi_{0} \in H_{\text {rad }}^{1}\left(\mathbb{R}^{2}\right),\left\|\phi_{0}\right\|_{L^{2}}=\left\|Q^{(0)}\right\|_{L^{2}}$ cannot blow up in finite time, as stated in Theorem 1.3.

Theorem 1.6 (Rigidity of blowup in infinite time in the self-dual case). For $m \in \mathbb{N}, g=1$ and initial data $\phi_{0} \in H_{m}^{1}\left(\mathbb{R}^{2}\right),\left\|\phi_{0}\right\|_{L^{2}}=\left\|Q^{(m)}\right\|_{L^{2}}$, if the solution of (eCSS) $\phi$ blows up in infinite time, say at $+\infty$, i.e. $\|\phi\|_{L_{t, x}^{4}\left([0,+\infty) \times \mathbb{R}^{2}\right)}=\infty$, then there exists $\gamma \in[0,2 \pi), \lambda \in$ $\mathbb{R}_{+}$such that

$$
\phi(t, r)=e^{i \gamma} \lambda \phi^{(m)}(\lambda r) .
$$

Remark 1.7. For $m \geq 1$, after restricting initial data to a smaller space $\Sigma:=\left\{f \in H_{m}^{1}\right.$ : $\left.|x| f \in L^{2}\right\}$, these two results are equivalent through pseudoconformal transformation (1.18), since pseudoconformal transformation maps $\Sigma$ into itself.

Since the threshold behavior also appears for the non-self-dual case $g>1$, we may expect similar rigidity for the threshold solution. Here we present the result for finite-time blowup.

Theorem 1.8 (Rigidity of blowup in finite time for $g>1$ ). For $m \in \mathbb{N}$ and $g>1$, if $\phi_{0} \in H_{m}^{1}\left(\mathbb{R}^{2}\right),\left\|u_{0}\right\|_{L^{2}}=c_{m, g}$ and the solution of (eCSS) $\phi$ blows up at $T>0$, then there exists $\gamma \in[0,2 \pi), \lambda \in \mathbb{R}_{+}$and an m-equivariant standing wave solution $\psi^{(m, g)}(t, x)=$ $e^{i \alpha t} \phi^{(m, g)}(x)(\alpha \geq 0)$ solving (eCSS), such that

$$
\phi(t, x)=e^{i \gamma} \mathrm{PC}_{T}\left[\lambda \psi^{(m, g)}\left(\lambda^{2} \cdot, \lambda \cdot\right)\right](t, x) \quad \forall t<T .
$$

Remark 1.9. Compared with Theorem 1.4, we do not know whether all the standing wave solutions are the same (up to symmetry). Also, due to the lack of knowledge on the uniqueness of the soliton and spectral analysis of its perturbation, our current approach for Theorem 1.6 cannot apply for the $g>1$ case.

Remark 1.10. In the non-self-dual case $g>1$, we only know $\mathrm{PC}_{T}\left[\psi^{(m, g, \alpha)}\right] \in H_{m}^{1}$ when $\alpha>0$ from the exponential decay in Proposition 2.2. For the $\alpha=0$ case we may not have 
$H_{m}^{1}$ finite-time blowup with solution $\mathrm{PC}_{T}\left[\psi^{(m, g, 0)}\right]$ as in Remark 1.5. But such a static solution is actually conjectured not to exist ([5]).

Equation (eCSS) with $g \geq 1$ can be viewed as a gauged version of the mass-critical focusing Schrödinger equation

$$
\left(i \partial_{t}+\Delta\right) u=-|u|^{\frac{4}{d}} u .
$$

It shares many essential features with (NLS) such as symmetries, conservation laws and soliton behaviors. So it is worthwhile reviewing the results of (NLS).

Equation (NLS) is also $L^{2}$-critical with pseudoconformal symmetry. It has a unique standing wave soliton $([3,37]) e^{i t} R(x)$, with $R(x)$ radial, positive and Schwartz, solving an elliptic equation

$$
\Delta R-R+R^{3}=0 .
$$

Weinstein ([54]) proved that any $H^{1}$ initial data with mass less than $\|R\|_{L^{2}}$ will generate a global solution. Killip-Tao-Visan ([32]) then showed global well-posedness and scattering for radial data with mass below the threshold, and the higher dimension case was solved by [33]. Finally, Dodson ([13]) extended this threshold result to general $L^{2}$ nonradial data for all dimensions.

Now we come to the threshold characterization results for (NLS). Combined with a virial argument and a rigidity result of Weinstein ([55]), Merle ([47,48]) proved the rigidity of blowup at finite time for $H^{1}$ data with threshold mass. The proof was simplified by Hmidi-Karaani ([21]) via profile decomposition. On infinite-time blowup, the first result is due to Killip-Li-Visan-Zhang ([31]), who showed that for $d \geq 4$ (and later for $d=2,3$ in [42]), $H^{1}$ radial data, a rigidity theorem like Theorem 1.6 holds. Li-Zhang then developed a local iteration scheme to obtain additional regularity in [40], which implies the rigidity result in $L^{2}\left(\mathbb{R}^{d}\right)$ for radial data as $d \geq 4$ ([40]), and later for splitting-spherical symmetry as $d=6$ ([39]). Our work is in a similar spirit to [31,42,48].

However, we should also point out some differences between (eCSS) and (NLS). Firstly, (NLS) admits a standing wave $e^{i t} R(x)$ with $R$ decaying exponentially, while ground state $Q$ for the self-dual (eCSS) is a static solution with only polynomial decay. Secondly, ground state $R$ for (NLS) serves as an extremizer of the Gagliardo-Nirenberg interpolation inequality, which is important in many of the compactness arguments, but we do not have such a characterization for $Q$. Besides, the nonlocal nonlinearity for (eCSS) makes the analysis more challenging, especially when we need to analyze the linearized operator around the ground state. On the other hand, we are lucky enough that the Bogomol'nyi operator and the self-duality structure of (eCSS) are of great help in overcoming the new difficulties and proving our results.

Finally, we would like to mention one more series of relevant results: characterization of the threshold solution for energy-critical equations. The pioneering work is attributed to Duyckaerts-Merle $([15,16])$. They characterized the threshold radial solution for $d=$ $3,4,5$ of the energy-critical wave equation and Schrödinger equation by detailed spectral analysis, the modulation method and concentration-compactness method. For subsequent related works, we refer to $[17,25,30,38,41,49]$. 


\subsection{Outline of the proof}

Our result consists of two parts: finite-time blowup (Theorems 1.4 and 1.8) and infinitetime blowup (Theorem 1.6). The starting point is the variational characterization of the ground state (see the elliptic theory in Section 2.3). Then the proofs bifurcate since they rely on very different strategies.

(1) Rigidity of finite-time blowup. The proof for the finite-time case follows the framework of [21], which serves as a simplification of Merle's original work ([47,48]). We start with a sequential rigidity result in Proposition 3.4. Specifically, if a sequence of threshold charge functions blows up in $H^{1}$ norm with energy bounded, then they converge to the soliton up to symmetry in $H^{1}$. For a solution $\phi$ blowing up at finite time $T$, this sequential rigidity implies that along a sequence of time there is charge concentration. We can then apply truncated virial identity and explore the relation of virial quantity with energy (2.10) to conclude $E\left(e^{i|x|^{2} /(4 T)} \phi_{0}\right)=0$, which forces $\phi$ to be the soliton after pseudoconformal transformation.

We remark that the Bogomol'nyi operator (2.4) and the $m$-equivariance condition help us to compensate for the lack of a sharp Gagliardo-Nirenberg inequality. In fact, our argument is even simpler than [21]. Also, the above argument works for both the self-dual and non-self-dual cases.

(2) Rigidity of infinite-time blowup. In this case, the proof is more complicated. The minimal blowup solution is characterized as having the compactness property, which is further illustrated as almost periodic modulo symmetry ([44]); see Theorem 4.8. This property indicates a uniform localization of charge. If we can further control the kinetic energy to be uniformly small near infinity (Theorem 4.11 ), we quickly reach a contradiction using a virial-type argument if the energy is positive.

So the main difficulty reduces to the proof of Theorem 4.11. We proceed in the spirit of [31] and its improvement [42]. In [31], Killip-Li-Visan-Zhang used the in-out decomposition, weighted Strichartz estimates and nonscattering Duhamel principle to prove the uniform localization of kinetic energy for minimal infinite-time blowup solutions of (NLS), for $d \geq 4$. The high power of nonlinearity causes trouble in low dimensions. To overcome the difficulty, Li-Zhang ([42]) used modulation analysis to prove a weaker localization theorem (similar to Proposition 4.12). This technique requires a good understanding of the spectral information for a linearized operator around ground state. For (eCSS), the linearized operator $\mathscr{L}_{Q}$ is nonlocal, which is usually difficult to analyze. Luckily the self-duality provides good structure, and the spectral information is carefully studied in [35]. Also there are more nonlocal terms to deal with, and that makes this part of the proof particularly long and complicated.

The structure of this article is as follows. In Section 2 we recall the Bogomol'nyi operator, elliptic theory and truncated virial estimation as preparation. Sections 3 and 4 deal with the finite-time case and the infinite-time case, respectively. Only the infinitetime blowup rigidity demands a great deal of harmonic analysis and spectral analysis for 
(eCSS), so we will record those tools therein. We remark that throughout the rest of this paper, we consider the non-self-dual case only in Section 3.3.

\section{Preliminaries}

\subsection{Notation}

Since we mainly work with $m$-equivariant functions $\phi(x)=e^{i m \theta} u(r): \mathbb{R}^{2} \rightarrow \mathbb{C}$, we usually refer to the radial part of such a function $\phi$ as $u$. And we will not distinguish them as acted on by functionals or operators, if there is no confusion. For example, $A_{\alpha}[u]:=A_{\alpha}[\phi], E[u]:=E[\phi, A[\phi]]$ and $\|u\|_{\dot{H}_{m}^{1}}:=\|f\|_{\dot{H}_{m}^{1}}=\|f\|_{\dot{H}^{1}}$.

We write $X \lesssim Y$ or $Y \gtrsim X$ to indicate $X \leq C Y$ for some constant $C>0$. If $C$ depends upon some additional parameters, we will indicate this with subscripts. For example, $X \lesssim_{\phi} Y$ means $X \leq C(\phi) Y$. We use $O(Y)$ to denote any quantity $X$ such that $|X| \lesssim Y$.

\subsection{Bogomol'nyi operator}

We first introduce the Bogomol'nyi operator

$$
\boldsymbol{D}_{+}:=D_{1}+i D_{2}=e^{i \theta}\left(\boldsymbol{D}_{r}+\frac{i}{r} \boldsymbol{D}_{\theta}\right)
$$

Then we have the Bogomol'nyi identity

$$
\left|\boldsymbol{D}_{x} \phi\right|^{2}=\left|\boldsymbol{D}_{+} \phi\right|^{2}+\nabla \times \boldsymbol{J}-F_{12}|\phi|^{2},
$$

where $\boldsymbol{J}:=\left(\operatorname{Im}\left(\bar{\phi} \boldsymbol{D}_{1} \phi\right), \operatorname{Im}\left(\bar{\phi} \boldsymbol{D}_{2} \phi\right)\right)$. Using Green's formula we can rewrite the energy functional as

$$
E[\phi]=\int_{\mathbb{R}^{2}}\left(\frac{1}{2}\left|\boldsymbol{D}_{x} \phi\right|^{2}-\frac{g}{4}|\phi|^{4}\right) d x=\int_{\mathbb{R}^{2}}\left(\frac{1}{2}\left|\boldsymbol{D}_{+} \phi\right|^{2}+\frac{1-g}{4}|\phi|^{4}\right) d x .
$$

Under the equivariant ansatz (1.7), the Bogomol'nyi operator takes the form

$$
\boldsymbol{D}_{+} \phi=\left[\left(\partial_{r}-\frac{m+A_{\theta}}{r}\right) u\right] e^{i(m+1) \theta}
$$

Taking the radial part, we also use $\boldsymbol{D}_{+}$to denote its action,

$$
\boldsymbol{D}_{+} u=\left(\partial_{r}-\frac{m+A_{\theta}}{r}\right) u \text {. }
$$

For the self-dual case $g=1$, the energy turns into

$$
E[\phi]=\int_{\mathbb{R}^{2}} \frac{1}{2}\left|\boldsymbol{D}_{+} \phi\right|^{2} d x .
$$

In this case, the minimizer of energy will satisfy a solvable first-order PDE $\boldsymbol{D}_{+} u=0$, implying the following variational characterization easily. 


\subsection{Variational characterization of the ground state}

We record the variational characterization of ground states of (eCSS) from elliptic theory, for both the self-dual case and the non-self-dual case. For completeness, we give their proofs in Appendix A.

Proposition 2.1 (Variational characterization in the self-dual case ([5,6,35])). Let $g=1$. For $\phi_{0} \in H_{m}^{1}\left(\mathbb{R}^{2}\right)-\{0\}$, we have $E\left[\phi_{0}\right] \geq 0$. Moreover, $E\left[\phi_{0}\right]=0$ if and only if $\phi_{0}$ is equal to $\phi^{(m)}$ in (1.17) up to $L^{2}$-scaling and phase rotation, which also implies that $\phi$ is a static solution of (eCSS).

Proposition 2.2 (Variational characterization in the non-self-dual case ([44])). Let $g>1$. For $\phi_{0} \in H_{m}^{1}-\{0\},\left\|\phi_{0}\right\|_{L^{2}} \leq c_{m, g}$, we have $E\left[\phi_{0}\right] \geq 0$. Moreover, if $E\left[\phi_{0}\right]=0$, then $\left\|\phi_{0}\right\|_{L^{2}}=c_{m, g}$ and there exists $\alpha \geq 0$ such that $\psi^{(m, g, \alpha)}(t, x)=e^{i \alpha t} \phi^{(m, g, \alpha)}(x)$ is a standing wave solution of (eCSS). We also know that any solution $\phi^{(m, g, \alpha)}$ decays exponentially for $\alpha>0$.

We remark that Proposition 2.2 is weaker than Proposition 2.1 by lack of uniqueness of the ground state. This accounts for the difference between Theorems 1.4 and 1.8.

\subsection{Truncated virial Identity}

The general virial identity for (CSS) is computed in [44]. We establish the truncated version through direct computation here.

Proposition 2.3 (Truncated virial identity). Let $\left(\phi, A_{0}, A_{1}, A_{2}\right)$ be a solution to (CSS), and $\chi \in C_{0, \mathrm{rad}}^{\infty}\left(\mathbb{R}^{2}\right)$. We have

$$
\begin{aligned}
\partial_{t} \int_{\mathbb{R}^{2}} \chi(r)|\phi|^{2} d x=2 \iint & \partial_{r} \chi \operatorname{Im}\left(\bar{\phi} \boldsymbol{D}_{r} \phi\right) r d r d \theta \\
\partial_{t}^{2} \int_{\mathbb{R}^{2}} \chi(r)|\phi|^{2} d x=2 \iint & 2 r \partial_{r} \chi\left|\boldsymbol{D}_{r} \phi\right|^{2}+\left[\frac{1}{r^{2}} \partial_{r}\left(r \partial_{r} \chi\right)-\partial_{r}\left(\frac{1}{r} \partial_{r} \chi\right)\right]\left|\boldsymbol{D}_{\theta} \phi\right|^{2} \\
& -\frac{1}{2} g \partial_{r}\left(r \partial_{r} \chi\right)|\phi|^{4} \\
+ & {\left[-\partial_{r}^{3}\left(r \partial_{r} \chi\right)+\frac{1}{2} \partial_{r}^{2} \partial_{r} \chi+\frac{1}{2} \partial\left(\frac{1}{r} \partial_{r} \chi\right)\right]|\phi|^{2} d r d \theta }
\end{aligned}
$$

Remark 2.4. In particular, when $\chi=|x|^{2}$ and $|x| \phi \in L^{2}$, a limiting argument implies the virial identity

$$
\begin{aligned}
& \partial_{t} \int_{\mathbb{R}^{2}}|x|^{2}|\phi|^{2}=4 \int_{\mathbb{R}^{2}} \operatorname{Im}\left(\bar{\phi} r D_{r} \phi\right), \\
& \partial_{t}^{2} \int_{\mathbb{R}^{2}}|x|^{2}|\phi|^{2}=16 E[\phi] .
\end{aligned}
$$

Via direct computation, the quadratic structure implies its cooperation with energy:

$$
8 t^{2} E\left[e^{i \frac{|x|^{2}}{4 t}} \phi(0)\right]=\int_{\mathbb{R}^{2}}|x|^{2}|\phi(t, x)|^{2} .
$$


Proof of Proposition 2.3. We define the stress-energy tensor

$$
T_{00}=F_{\theta r}=\frac{1}{2} r|\phi|^{2}, \quad T_{0 r}=F_{0 \theta}=r \operatorname{Im}\left(\bar{\phi} \boldsymbol{D}_{r} \phi\right), \quad T_{0 \theta}=F_{r 0}=\frac{1}{r} \operatorname{Im}\left(\bar{\phi} \boldsymbol{D}_{\theta} \phi\right) .
$$

From $d F=d^{2} A=0$ we have

$$
\partial_{\alpha} T_{0 \alpha}=0
$$

Recall ([44, Lemma 5.1])

$$
\begin{aligned}
\partial_{t} T_{0 r} & =-\left(2+2 r \partial_{r}\right)\left|\boldsymbol{D}_{r} \phi\right|^{2}+\frac{1}{2} r g \partial_{r}|\phi|^{4} \\
& +\frac{1}{2} \partial_{r}\left|\boldsymbol{D}_{\theta} \phi\right|^{2}-\frac{2}{r} \partial_{\theta} \operatorname{Re}\left(\overline{\boldsymbol{D}_{\theta} \phi} \boldsymbol{D}_{r} \phi\right) \\
& +r \partial_{r}\left[\frac{1}{r^{2}}\left(\frac{1}{2} \partial_{\theta}^{2}|\phi|^{2}-\left|\boldsymbol{D}_{\theta} \phi\right|^{2}\right)\right]+\left(\frac{1}{2} r \partial_{r}^{3}+\frac{1}{2} \partial_{r}^{2}-\frac{1}{2 r} \partial_{r}\right)|\phi|^{2} .
\end{aligned}
$$

Now we apply (2.11) to show (2.6):

$$
\begin{aligned}
\partial_{t} \int_{\mathbb{R}^{2}} \chi(r)|\phi|^{2} d x & =2 \iint \chi(r) \partial_{t} T_{00} d r d \theta \\
& =-2 \iint \chi(r)\left(\partial_{r} T_{0 r}+\partial_{\theta} T_{0 \theta}\right) d r d \theta=2 \iint \partial_{r} \chi T_{0 r} d r d \theta .
\end{aligned}
$$

Next we can invoke (2.12) and take the derivative of time again to get (2.7).

If we take $\chi$ to be a smooth truncation of $|x|^{2}$, the computation within $\{|x| \leq R\}$ remains the same as the standard virial identity (2.9). The following estimates follow immediately.

Corollary 2.5. Let $\left(\phi, A_{0}, A_{1}, A_{2}\right)$ be a solution to (CSS), and $\chi_{R}=R^{2} \chi\left(R^{-1} \cdot\right)$ is the smooth cutoff of $|x|^{2}$, with $\chi \in C_{0, \text { rad }}^{\infty}(\{|x| \leq 2\})$ and

$$
\chi(x)=\chi(|x|)= \begin{cases}|x|^{2}, & |x|<1 \\ 0, & |x| \geq 2\end{cases}
$$

Then

$$
\begin{aligned}
\partial_{t} \int_{\mathbb{R}^{2}} \chi_{R}|\phi|^{2}= & 2 \int_{\mathbb{R}^{2}} \partial_{r} \chi_{R} \operatorname{Im}\left(\bar{\phi} \boldsymbol{D}_{r} \phi\right), \\
\partial_{t}^{2} \int_{\mathbb{R}^{2}} \chi_{R}|\phi|^{2}= & 16 E[\phi]+O\left(\frac{1}{R^{2}} \int_{|x| \geq R}|\phi|^{2}\right) \\
& +O\left(\int_{|x| \geq R}|\nabla \phi|^{2}+\int_{|x| \geq R}|\phi|^{4}\right) .
\end{aligned}
$$

Remark 2.6. By constructing $\chi$ through convolution, it is easy to verify that it satisfies

$$
\left|\nabla \chi_{R}(x)\right|^{2} \lesssim \chi_{R}(x) .
$$




\section{Finite-time blowup}

In this section we prove Theorems 1.4 and 1.8. We first consider the self-dual case, and then the non-self-dual case with a small modification.

\subsection{Rigidity of a normalized sequence}

We show the rigidity of a normalized $H^{1}$ blowup sequence.

Recall

$$
\begin{gathered}
\left\|\boldsymbol{D}_{x} f\right\|_{L^{2}}^{2}=\left\|D_{r} f\right\|_{L^{2}}^{2}+\left\|D_{\theta} f\right\|_{L^{2}}^{2}=\left\|\partial_{r} f\right\|_{L^{2}}^{2}+\left\|\frac{m+A_{\theta}[f]}{r} f\right\|_{L^{2}}^{2}, \\
E[f]=\frac{1}{2}\left\|\boldsymbol{D}_{+} f\right\|_{L^{2}}^{2}=\frac{1}{2}\left\|\boldsymbol{D}_{x} f\right\|_{L^{2}}^{2}-\frac{1}{4}\|f\|_{L^{4}}^{4}
\end{gathered}
$$

and the scaling property

$$
\left\|\boldsymbol{D}_{x}(\lambda f(\lambda \cdot))\right\|_{L^{2}}^{2}=\lambda^{2}\left\|\boldsymbol{D}_{x} f\right\|_{L^{2}}^{2} \quad \forall \lambda \in \mathbb{R}_{+} .
$$

To begin with, we discuss some properties of $\left\|\boldsymbol{D}_{x} f\right\|_{L^{2}}^{2}$, showing it is to some extent equivalent to the $\dot{H}_{m}^{1}$ norm. The first two lemmas are concerned with size and weak convergence.

Lemma 3.1. For $m \geq 1, f \in H_{m}^{1}$,

$$
\begin{gathered}
\left\|\frac{1}{r} f\right\|_{L^{2}} \lesssim_{m,\|f\|_{L^{2}}}\left\|\frac{m+A_{\theta}[f]}{r} f\right\|_{L^{2}}, \\
\left\|\frac{A_{\theta}[f]}{r} f\right\|_{L^{2}} \lesssim\|f\|_{L^{2}}^{2}\left\|\partial_{r} f\right\|_{L^{2}} .
\end{gathered}
$$

As a consequence, for $m \geq 0, f \in H_{m}^{1}$,

$$
\|f\|_{\dot{H}_{m}^{1}\left(\mathbb{R}^{2}\right)}^{2} \sim_{m,\|f\|_{L^{2}}}\left\|\boldsymbol{D}_{x} f\right\|_{L^{2}}^{2} .
$$

Lemma 3.2. For $m \geq 0, f_{n}$ and $f$ uniformly bounded in $H_{m}^{1}\left(\mathbb{R}^{2}\right)$ and $f_{n} \rightarrow f$ in $H^{1}$, we have

$$
\begin{array}{rlrl}
\partial_{r} f_{n} & \rightarrow \partial_{r} f & & \text { in } L^{2}, \\
\frac{1}{r} A_{\theta}\left[f_{n}\right] f_{n} & \rightarrow \frac{1}{r} A_{\theta}[f] f & \text { in } L^{2}
\end{array}
$$

and, for $m \geq 1$,

$$
\frac{1}{r} f_{n} \rightarrow \frac{1}{r} f \quad \text { in } L^{2} .
$$

In particular,

$$
\boldsymbol{D}_{+} f_{n}=\partial_{r} f_{n}-\frac{m+A_{\theta}\left[f_{n}\right]}{r} f_{n} \rightarrow \boldsymbol{D}_{+} f \quad \text { in } L^{2}
$$


The following lemma indicates that we can recover strong convergence in $H^{1}$ through $L^{2}$ convergence for $v_{n}$ and $\boldsymbol{D}_{+} v_{n}$.

Lemma 3.3. Suppose we have $\left\{v_{n}\right\}$ and $v$ uniformly bounded in $H_{m}^{1}$, and

$$
\begin{gathered}
2 v_{n} \rightarrow v \text { in } H^{1}, \quad v_{n} \rightarrow v \text { in } L^{2}, \\
\boldsymbol{D}_{+} v_{n} \rightarrow \boldsymbol{D}_{+} v \text { in } L^{2}, \quad v_{n} \rightarrow v \text { in } L^{4} .
\end{gathered}
$$

Then we will have

$$
v_{n} \rightarrow v \text { in } H^{1}
$$

We leave their proof to Appendix B. Now we work on the rigidity of the $H^{1}$ blowup sequence. Take a time sequence of the $H^{1}$ blowup solution. By rescaling, we normalize the charge and $\dot{H}^{1}$ norm with the energy going to zero. The proposition shows that such a sequence must converge to the ground state.

Proposition 3.4. For $m \geq 0$, let $v_{n} \in H_{m}^{1}\left(\mathbb{R}^{2}\right)$, satisfying

$$
\begin{gathered}
\left\|v_{n}\right\|_{L^{2}}=\left\|\phi^{(m)}\right\|_{L^{2}}, \quad\left\|v_{n}\right\|_{\dot{H}_{m}^{1}}=\left\|\phi^{(m)}\right\|_{\dot{H}_{m}^{1}}, \\
E\left[v_{n}\right]=\frac{1}{2}\left\|\boldsymbol{D}_{+} v_{n}\right\|_{L^{2}}^{2} \rightarrow 0 \quad \text { as } n \rightarrow \infty,
\end{gathered}
$$

where $\phi^{(m)}=Q e^{i m \theta} \in H_{m}^{1}$ is the static soliton. Then there exists $\gamma \in[0,2 \pi)$ and a subsequence of $\left\{v_{n}\right\}$ (still denoted by $\left\{v_{n}\right\}$ ) such that

$$
v_{n} \rightarrow e^{i \gamma} \phi^{(m)} \quad \text { in } H^{1}
$$

The following corollary is another equivalent way of stating this rigidity, which comes easily after a contradiction argument. It will be useful in modulation analysis to control the $H_{m}^{1}$ norm by energy (see Lemma 4.19).

Corollary 3.5. For $m \geq 0$, let $f \in H_{m}^{1}\left(\mathbb{R}^{2}\right)$, satisfying

$$
\|f\|_{L^{2}}=\left\|\phi^{(m)}\right\|_{L^{2}}, \quad\|f\|_{\dot{H}_{m}^{1}}=\left\|\phi^{(m)}\right\|_{\dot{H}_{m}^{1}} .
$$

Then the energy $E[f] \geq 0$ and, for any $\varepsilon>0$, there exists $\delta>0$ such that if $E[f]<\delta$, then there exists $\gamma \in[0,2 \pi)$ such that

$$
\left\|\phi^{(m)}-e^{i \gamma} f\right\|_{H_{m}^{1}}<\varepsilon .
$$

Proof of Proposition 3.4. The sequence $\left\{v_{n}\right\}$ is uniformly bounded in $H_{m}^{1}$, so we can extract a weakly convergent subsequence (still denoted by $\left\{v_{n}\right\}$ ) and $v \in H_{m}^{1}$,

$$
v_{n} \rightarrow v \quad \text { in } H^{1} \text {. }
$$

Since $H_{\text {rad }}^{1}\left(\mathbb{R}^{2}\right)$ compactly embeds in $L_{\text {rad }}^{p}\left(\mathbb{R}^{2}\right)$ for any $p \in(2, \infty)$, it is easy to see $H_{m}^{1}\left(\mathbb{R}^{2}\right)$ also compactly embeds in $L_{m}^{p}\left(\mathbb{R}^{2}\right)$. Hence

$$
v_{n} \rightarrow v \quad \text { in } L_{m}^{4}
$$


Note that the normalization condition and Lemma 3.1 imply

$$
\left\|\boldsymbol{D}_{x} v_{n}\right\|_{L^{2}}^{2} \gtrsim_{m}\left\|v_{n}\right\|_{\dot{H}_{m}^{1}}^{2}=\|Q\|_{\dot{H}_{m}^{1}}^{2} .
$$

So

$$
\|v\|_{L^{4}}=\lim _{n \rightarrow \infty}\left\|v_{n}\right\|_{L^{4}}=\lim _{n \rightarrow \infty}\left(-4 E\left[v_{n}\right]+2\left\|\boldsymbol{D}_{x} v_{n}\right\|_{L^{2}}^{2}\right) \gtrsim_{m}\|Q\|_{\dot{H}_{m}^{1}}^{2}>0 .
$$

Also, from Lemma 3.2, we have

$$
E[v]=\frac{1}{2}\left\|\boldsymbol{D}_{+} v\right\|_{L^{2}}^{2} \leq \liminf _{n \rightarrow \infty} \frac{1}{2}\left\|\boldsymbol{D}_{+} v_{n}\right\|_{L^{2}}^{2}=0 .
$$

Note that (3.12) ensures that $v$ is a nonzero function. Proposition 2.1 implies that $v$ is ground state up to symmetry. Namely, there exists $\gamma \in[0,2 \pi), \lambda \in \mathbb{R}_{+}$, such that

$$
v=e^{i \gamma} \lambda Q(\lambda r) e^{i m \theta}
$$

Hence

$$
\|v\|_{L^{2}}=\|Q\|_{L^{2}}=\left\|v_{n}\right\|_{L^{2}} .
$$

Together with the norm convergence of $\boldsymbol{D}_{+} v_{n}$, we now have two more strong convergences,

$$
\begin{array}{cc}
\boldsymbol{D}_{+} v_{n} \rightarrow \boldsymbol{D}_{+} v & \text { in } L^{2}, \\
v_{n} \rightarrow v & \text { in } L^{2} .
\end{array}
$$

From (3.10), (3.11), (3.15) and (3.16), we conclude $v_{n} \rightarrow v$ in $\dot{H}_{m}^{1}$ through Lemma 3.3, which also indicates the scaling parameter $\lambda=1$, thus completing the proof.

\subsection{Proof of Theorem 1.4}

First we need a Cauchy-Schwarz-type estimate. This estimate was first introduced by Banica for (NLS) in [1], and used in the proof of [21] to derive a crucial ODE control of truncated virial identity. It still holds for general $H^{1}$ functions even without the equivariant assumption.

Lemma 3.6 (Cauchy-Schwarz-type estimate). For $f \in H^{1}$ and all $R>0$, we have

$$
\left|\int \partial_{r} \chi_{R} \operatorname{Im}\left(\bar{f} \boldsymbol{D}_{r} f\right) d x\right| \leq\left(2 E[f] \int|f|^{2}\left|\partial_{r} \chi_{R}\right|^{2} d x\right)^{\frac{1}{2}},
$$

where $E[f]=\frac{1}{2}\left\|\boldsymbol{D}_{+} f\right\|_{L^{2}}^{2}$ is the energy for (CSS) in the self-dual case.

Proof. Notice that energy has another expression in polar coordinates (1.6) with $g=1$. So, with positivity of energy, for any $\alpha \in \mathbb{R}$,

$$
\begin{aligned}
0 \leq E\left[e^{i \alpha \chi_{R}(x)} f\right] & =\frac{1}{2} \int\left|\boldsymbol{D}_{r}\left(e^{i \alpha \chi_{R}} f\right)\right|^{2}+\frac{1}{r^{2}}\left|\boldsymbol{D}_{\theta}\left(e^{i \alpha \chi_{R}} f\right)\right|^{2}-\frac{1}{2}\left|e^{i \alpha \chi_{R}} f\right|^{4} d x \\
& =\frac{1}{2} \int\left|\boldsymbol{D}_{r} f+i \alpha \partial_{r} \chi_{R} f\right|^{2}+\frac{1}{r^{2}}\left|\boldsymbol{D}_{\theta} f\right|^{2}-\frac{1}{2}|f|^{4} d x \\
& =\left.\frac{\alpha^{2}}{2} \int|f|^{2}\left|\partial_{r} \chi_{R}\right|^{2}\right|^{2} d x-\alpha \int \partial_{r} \chi_{R} \operatorname{Im}\left(\bar{f} \boldsymbol{D}_{r} f\right) d x+E[f] .
\end{aligned}
$$


The positivity of this quadratic form implies a negative discriminant, which is exactly (3.17).

Now we are ready to prove Theorem 1.4.

Proof of Theorem 1.4. We divide the proof into two steps.

Step 1: Normalized rigidity and charge concentration behavior. From Sobolev embedding and regularity $H_{m}^{1}$, we see that the finite-time blowup condition

$$
\|\phi\|_{L_{t, x}^{4}\left([0, T) \times \mathbb{R}^{2}\right)}=\infty
$$

implies the $L_{t}^{\infty} H_{x}^{1}$ blowup. Namely, there exists a sequence of time $t_{n} \nearrow T$ such that

$$
\left\|\phi\left(t_{n}\right)\right\|_{H_{m}^{1}} \rightarrow \infty \quad \text { as } n \rightarrow \infty
$$

We set

$$
\rho_{n}=\frac{\left\|\phi^{(m)}\right\|_{\dot{H}_{m}^{1}}}{\left\|\phi\left(t_{n}, \cdot\right)\right\|_{\dot{H}_{m}^{1}}} \quad \text { and } \quad v_{n}=\rho_{n} \phi\left(t_{n}, \rho_{n} x\right) .
$$

Then from (3.18) and charge conservation, $\rho_{n} \rightarrow 0$ as $n \rightarrow \infty$. The sequence $\left\{v_{n}\right\}$ then satisfies

$$
\left\|v_{n}\right\|_{L^{2}}=\|Q\|_{L^{2}}, \quad\left\|v_{n}\right\|_{\dot{H}_{m}^{1}}=\left\|\phi^{(m)}\right\|_{\dot{H}_{m}^{1}} .
$$

Furthermore, by conservation of the energy,

$$
E\left[v_{n}\right]=\rho_{n}^{2} E\left[\phi_{0}\right] \rightarrow 0 \quad \text { as } n \rightarrow \infty .
$$

Hence, $\left\{v_{n}\right\}$ satisfies the assumption of Proposition 3.4, and we have

$$
e^{-i \gamma} \rho_{n} \phi\left(t_{n}, \rho_{n} x\right)=e^{-i \gamma} v_{n} \rightarrow Q \quad \text { in } H^{1} \text { as } n \rightarrow \infty,
$$

for some $\gamma \in[0,2 \pi)$.

This easily implies the charge concentration behavior

$$
\left|\phi\left(t_{n}, x\right)\right|^{2}-\|Q\|_{L^{2}}^{2} \delta_{x=0} \rightarrow 0 \quad \text { in } \mathscr{D}^{\prime}\left(\mathbb{R}^{2}\right),
$$

where $\mathscr{D}^{\prime}\left(\mathbb{R}^{2}\right)$ is the distribution on $\mathbb{R}^{2}$ and $\delta_{x=0}$ is the delta functional.

Step 2: Truncated virial estimate. Denote the truncated virial quantity for $\phi$ to be

$$
V_{R}(t)=\int \chi_{R}(x)|\phi(t, x)|^{2} d x
$$

where $\chi_{R}$ is the smooth truncation of $|x|^{2}$ as in Corollary 2.5. Putting Lemma 3.6 together with the bound (2.15), for $t \in[0, T)$ we get

$$
\begin{aligned}
\left|\partial_{t} V_{R}(t)\right| & =2\left|\int \partial_{r} \chi_{R} \operatorname{Im}\left(\phi \overline{(t)} \boldsymbol{D}_{r} \phi(t)\right) d x\right| \leq 2\left(2 E[\phi(t)] \int|\phi(t)|^{2}\left|\partial_{r} \chi_{R}\right|^{2} d x\right)^{\frac{1}{2}} \\
& \leq C E\left[\phi_{0}\right]^{\frac{1}{2}}\left(\int|\phi(t)|^{2} \chi_{R} d x\right)^{\frac{1}{2}} \lesssim_{\phi_{0}}\left(V_{R}(t)\right)^{\frac{1}{2}} .
\end{aligned}
$$


By integration we obtain, for every $t \in[0, T)$,

$$
\left|\left(V_{R}(t)\right)^{\frac{1}{2}}-\left(V_{R}\left(t_{n}\right)\right)^{\frac{1}{2}}\right| \leq C\left(\phi_{0}\right)\left|t_{n}-t\right| .
$$

Now take $n \rightarrow \infty$, and from (3.20) we get

$$
\left|V_{R}(t)\right| \leq C\left(\phi_{0}\right)(T-t)^{2} .
$$

Noting that this bound is independent of $R$, by taking $R \rightarrow \infty$ we can see that the virial quantity is also controlled by the same bound. Hence (2.10) provides us with

$$
8 t^{2} E\left[e^{i \frac{|x|^{2}}{4 t}} \phi(0)\right]=\int_{\mathbb{R}^{2}}|x|^{2}|\phi(t, x)|^{2} \leq C\left(\phi_{0}\right)(T-t)^{2} .
$$

Let $t \nearrow T$; we get

$$
E\left[e^{i \frac{|x|^{2}}{4 T}} \phi_{0}\right]=0
$$

Thus Proposition 2.1 indicates that there exists $\gamma \in[0,2 \pi), \lambda \in \mathbb{R}_{+}$, such that

$$
\phi_{0}=e^{i \gamma} \lambda e^{-i \frac{|x|^{2}}{4 T}} Q(\lambda r) e^{i m \theta}=e^{i \gamma} \mathrm{PC}_{T}\left[\tilde{\lambda} \phi^{(m)}(\tilde{\lambda} \cdot)\right](0, r),
$$

with $\tilde{\lambda}=\lambda T$. This concludes the proof of Theorem 1.4.

\subsection{Non-self-dual case}

In this subsection we prove Theorem 1.8 via the same strategy as the self-dual case.

Proposition 3.7. For $m \geq 0, g>1$, let $v_{n} \in H_{m}^{1}\left(\mathbb{R}^{2}\right)$, satisfying

$$
\begin{gathered}
\left\|v_{n}\right\|_{L^{2}}=c_{m, g}, \quad\left\|v_{n}\right\|_{\dot{H}^{1}}=M, \\
E\left[v_{n}\right]=\frac{1}{2}\left\|\boldsymbol{D}_{+} v_{n}\right\|_{L^{2}}^{2}-\frac{g-1}{4}\left\|v_{n}\right\|_{L^{4}}^{4} \rightarrow 0 \quad \text { as } n \rightarrow \infty .
\end{gathered}
$$

Then there exists $\gamma \in[0,2 \pi)$, a subsequence of $\left\{v_{n}\right\}$ (still denoted by $\left\{v_{n}\right\}$ ) and $v \in H_{m}^{1}$ such that

$$
v_{n} \rightarrow v \quad \text { in } H^{1}
$$

and $\psi(t, x)=e^{i \alpha t} v(x)$ for some $\alpha \in \mathbb{R} \backslash\{0\}$ is a standing wave solution of (eCSS).

Proof. By uniform boundedness in the $H^{1}$ norm and compact embedding, we know there exists $v \in H_{m}^{1}$ such that

$$
v_{n} \rightarrow v \text { in } H^{1}, \quad v_{n} \rightarrow v \text { in } L^{4}
$$

Again, by the lower bound of $\left\|\boldsymbol{D}_{x} v_{n}\right\|_{L^{2}}$ from Lemma 3.1 and $L^{4}$ convergence, we know $v$ is nontrivial. And from Lemma 3.2,

$$
E[v]=\frac{1}{2}\left\|\boldsymbol{D}_{+} v\right\|_{L^{2}}^{2}-\frac{g-1}{4}\|v\|_{L^{4}}^{4} \leq \liminf _{n \rightarrow \infty} \frac{1}{2}\left\|\boldsymbol{D}_{+} v_{n}\right\|_{L^{2}}^{2}-\frac{g-1}{4}\left\|v_{n}\right\|_{L^{4}}^{4}=0 .
$$


Note that

$$
\|v\|_{L^{2}} \leq \liminf _{n \rightarrow \infty}\left\|v_{n}\right\|_{L^{2}}=c_{m, g}
$$

and Proposition 2.2 forces $E[v] \geq 0$. So $E[v]=0$ and hence $v$ is a standing wave solution to (eCSS) with critical charge $c_{m, g}$. The norm convergence of (3.23) and (3.24) implies that

$$
v_{n} \rightarrow v \text { in } L^{2}, \quad \boldsymbol{D}_{+} v_{n} \rightarrow \boldsymbol{D}_{+} v \text { in } L^{2} .
$$

Now Lemma 3.3 ensures the $H^{1}$ convergence and completes the proof.

Since the positivity of energy holds for $\|f\|_{L^{2}} \leq c_{m, g}$, we can prove the counterpart of the Cauchy-Schwarz-type estimate for the non-self-dual case in the same way as Lemma 3.6. We state the lemma and omit its proof.

Lemma 3.8. Fix $m \geq 0$ and $g>1$. For $f \in H^{1},\|f\|_{L^{2}} \leq c_{m, g}$ and all $R>0$, we have

$$
\left|\int \partial_{r} \chi_{R} \operatorname{Im}\left(\bar{f} \boldsymbol{D}_{r} f\right) d x\right| \leq\left(2 E[f] \int|f|^{2}\left|\partial_{r} \chi_{R}\right|^{2} d x\right)^{\frac{1}{2}},
$$

where $E[f]=\frac{1}{2}\left\|\boldsymbol{D}_{+} u\right\|_{L^{2}}^{2}-\frac{g-1}{4}\|f\|_{L^{4}}^{4}$ is the energy for (CSS) in the non-self-dual case.

Then we finish the proof of Theorem 1.8 by the same argument as Theorem 1.4, using a charge concentration argument and truncated virial estimate.

\section{Infinite-time blowup}

In this section we prove Theorem 1.6. First, in Section 4.1 we introduce several tools from harmonic analysis, including the concentration compactness Proposition 4.8. Then we reduce the whole proof to a frequency decay estimate (Proposition 4.10) in Section 4.2 and prove that estimate in Sections 4.3 and 4.4.

\subsection{Preliminary on harmonic analysis}

4.1.1. Basic harmonic analysis. We introduce the Littlewood-Paley multipliers in the usual way. In particular, let $\varphi \in C_{0 \text {,rad }}^{\infty}(\{|x| \leq 2\})$ and

$$
\varphi(x)=\varphi(|x|)= \begin{cases}1, & |x|<1 \\ 0, & |x| \geq 2\end{cases}
$$

and its scaling for $R>0$,

$$
\varphi_{\leq R}:=\varphi\left(R^{-1} \cdot\right), \quad \varphi_{>R}:=1-\varphi_{\leq R} .
$$

Then for each $N \in 2^{\mathbb{Z}}$, define

$$
\mathscr{F}\left(P_{\leq N} f\right)(\xi):=\varphi_{\leq N}(|\xi|) \hat{f}(\xi), \quad P_{>N}=1-P_{\leq N}, \quad P_{N}=P_{\leq N}-P_{\leq \frac{N}{2}}
$$


and the fattened Littlewood-Paley operators

$$
\widetilde{P}_{N}:=P_{N / 2}+P_{N}+P_{2 N}
$$

The basic estimate in Littlewood-Paley theory is the following Bernstein estimate.

Lemma 4.1 (Bernstein estimates). For $1 \leq p \leq q \leq \infty$,

$$
\begin{aligned}
\left\||\nabla|^{ \pm s} P_{N} f\right\|_{L^{p}\left(\mathbb{R}^{d}\right)} & \sim N^{ \pm s}\left\|P_{N} f\right\|_{L^{p}\left(\mathbb{R}^{d}\right)}, \\
\left\|P_{\leq N} f\right\|_{L^{p}\left(\mathbb{R}^{d}\right)} & \lesssim N^{\frac{d}{p}-\frac{d}{q}}\left\|P_{\leq N} f\right\|_{L^{p}\left(\mathbb{R}^{d}\right)}, \\
\left\|P_{N} f\right\|_{L^{p}\left(\mathbb{R}^{d}\right)} & \lesssim N^{\frac{d}{p}-\frac{d}{q}}\left\|P_{N} f\right\|_{L^{p}\left(\mathbb{R}^{d}\right)} .
\end{aligned}
$$

While it is true that spatial cutoffs do not commute with Littlewood-Paley operators, the commutator is not "too bad", so that a weaker form of almost orthogonality still holds.

Lemma 4.2 (Mismatch estimates in physical space ([31])). Let $R, N>0$. Then

$$
\begin{gathered}
\left\|\varphi_{>R} P_{\leq N} \varphi_{\leq \frac{R}{2}} f\right\|_{L^{p}} \lesssim_{m} N^{-m} R^{-m}\|f\|_{L^{p}}, \\
\left\|\varphi_{>R} \nabla P_{\leq N} \varphi_{\leq \frac{R}{2}} f\right\|_{L^{p}} \lesssim_{m} N^{1-m} R^{-m}\|f\|_{L^{p}}
\end{gathered}
$$

for any $1 \leq p \leq \infty$ and $m \geq 0$.

Similar estimates hold when the roles of frequency and physical spaces are interchanged.

Lemma 4.3 (Mismatch estimate in frequency space ([31])). For $R>0$ and $N, M>0$ such that $\max \{N, M\} \geq 4 \min \{N, M\}$,

$$
\begin{gathered}
\left\|P_{N} \varphi_{\leq R} P_{M} f\right\|_{L^{2}} \lesssim_{m} \max \{N, M\}^{-m} R^{-m}\|f\|_{L^{2}}, \\
\left\|P_{N} \varphi_{\leq R} \nabla P_{M} f\right\|_{L^{2}} \lesssim_{m} M \max \{N, M\}^{-m} R^{-m}\|f\|_{L^{2}},
\end{gathered}
$$

for any $m \geq 0$. The same estimates hold if we replace $\varphi_{\leq R}$ by $\varphi_{>R}$, or $P_{N}$ by $P_{<N}$ when $M \geq 4 N$.

4.1.2. Strichartz estimates. We present the classical linear Strichartz estimates. See for example [7].

Lemma 4.4 (Strichartz estimates). Let I be a time interval with $0 \in I$ and $u(0)=u_{0} \in L^{2}$ and $F \in L_{t}^{\frac{4}{3}} L_{x}^{\frac{4}{3}}\left(I \times \mathbb{R}^{2}\right)$. Then the strong solution $u$ to the linear Schrödinger equation

$$
u(t):=e^{i t \Delta} u_{0}-i \int_{0}^{t} e^{i\left(t-t^{\prime}\right) \Delta} F\left(t^{\prime}\right) d t^{\prime}, \quad t \in I,
$$

satisfies the estimate

$$
\|u\|_{L_{t}^{\infty} L_{x}^{2}\left(I \times \mathbb{R}^{2}\right)}+\|u\|_{L_{t, x}^{4}\left(I \times \mathbb{R}^{2}\right)} \lesssim\left\|u_{0}\right\|_{L_{x}^{2}}+\|F\|_{L_{t, x}^{\frac{4}{3}}\left(I \times \mathbb{R}^{2}\right)} .
$$


The following weighted Strichartz estimate, which originates from the radial case ([32]), exploits the equivariance property heavily to obtain spatial decay.

Lemma 4.5 (Weighted Strichartz estimates). For $m \geq 0$, let $I$ be an interval, $t_{0} \in I$, and let $F: I \times \mathbb{R}^{2} \rightarrow \mathbb{C}$ be $m$-equivariant in space. Then

$$
\left\|\int_{t_{0}}^{t} e^{i\left(t-t^{\prime}\right) \Delta} F\left(t^{\prime}\right) d t^{\prime}\right\|_{L_{x}^{2}} \lesssim\left\||x|^{-\frac{1}{2}} F\right\|_{L_{t}^{\frac{4}{3}} L_{x}^{1}} .
$$

Proof. As in the standard proof of Strichartz estimates, besides the $T T^{*}$ method and the Hardy-Littlewood-Sobolev inequality, we only need to show a dispersive estimate

$$
\left\||x|^{\frac{1}{2}} e^{i t \Delta}|x|^{\frac{1}{2}} f\right\|_{L_{x}^{\infty}\left(\mathbb{R}^{2}\right)} \lesssim|t|^{-\frac{1}{2}}\|f\|_{L_{x}^{1}\left(\mathbb{R}^{2}\right)}
$$

for any $m$-equivariant function $f(x)=u(r) e^{i m \theta} \in L_{m}^{1}\left(\mathbb{R}^{2}\right)$. And this follows from the computation of the kernel of $e^{i t \Delta}$ applied to $m$-equivariant functions. Under polar coordinates $x:=(r \cos \theta, r \sin \theta), y:=(\rho \cos \alpha, \rho \sin \alpha)$, we see

$$
\begin{aligned}
\left(e^{i t \Delta} f\right)(y) & =\frac{1}{4 \pi i t} \int_{\mathbb{R}^{2}} f(x) e^{\frac{i|x-y|^{2}}{4 t}} d x \\
& =\frac{1}{4 \pi i t} \int_{0}^{\infty} u(r) \int_{0}^{2 \pi} e^{i m \theta} e^{i \frac{r^{2}+\rho^{2}-2 r \rho \cos (\theta-\alpha)}{4 t}} d \theta r d r \\
& =\frac{1}{4 \pi i t} e^{i m \alpha} \int_{0}^{\infty} u(r) e^{i \frac{r^{2}+\rho^{2}}{4 t}} \int_{0}^{2 \pi} e^{i\left(m \omega-\frac{r \rho}{2 t} \cos \omega\right)} d \omega r d r .
\end{aligned}
$$

That is, for radial functions, $e^{-i m \alpha} e^{i t \Delta} e^{i m \theta}$ has the kernel

$$
\begin{aligned}
{\left[e^{-i m \alpha} e^{i t \Delta} e^{i m \theta}\right](x, y) } & =\frac{1}{4 \pi i t} e^{i \frac{r^{2}+\rho^{2}}{4 t}} \int_{0}^{2 \pi} e^{i\left(m \omega-\frac{r \rho}{2 t} \cos \omega\right)} \frac{d \omega}{2 \pi} \\
& =\frac{1}{4 \pi i t} e^{i \frac{r^{2}+\rho^{2}}{4 t}} J_{m}\left(\frac{r \rho}{2 t}\right),
\end{aligned}
$$

where $J_{v}$ denotes the Bessel function of order $v$. So by the behavior of Bessel functions ([20]), we have $|(4.2)| \lesssim|r \rho t|^{-\frac{1}{2}}$. Inequality (4.1) follows immediately.

4.1.3. In-out decomposition. Finally, we present one more useful tool in the following analysis - the incoming/outgoing decomposition developed in [32,33], and the $m$ equivariant version in [44]. It derives from the relationship between Bessel functions and the Fourier transform of a radial function. For $f(r, \theta)=e^{i m \theta} u(r) \in L_{m}^{2}$,

$$
\hat{f}(\rho, \alpha)=2 \pi(-i)^{m} e^{i m \alpha} \int_{0}^{\infty} J_{m}(\rho r) f(r) r d r,
$$

where $J_{v}$ denotes the Bessel function of order $v$. We split the Bessel function $J_{m}$ into two Hankel functions, $H_{m}^{(1)}$ and $H_{m}^{(2)}$, corresponding to projections onto outgoing and incoming waves. In particular,

$$
J_{m}(|x||\xi|)=\frac{1}{2} H_{m}^{(1)}(|x||\xi|)+\frac{1}{2} H_{m}^{(2)}(|x||\xi|),
$$


where $H_{m}^{(1)}$ is the order $m$ Hankel function of the first kind and $H_{m}^{(2)}$ is the order $m$ Hankel function of the second kind. We can define the in-out decomposition ${ }^{7}$

$$
\begin{aligned}
{\left[P^{+} f\right](x) } & :=\frac{1}{4 \pi^{2}} e^{i m \theta} \int_{\mathbb{R}^{2}} H_{m}^{(1)}(|x||\xi|) J_{m}(|\xi||y|) f(|y|) d \xi d y \\
& =\frac{1}{2} f(x)+\frac{i}{2 \pi^{2}} \int_{\mathbb{R}^{2}}\left|\frac{y}{x}\right|^{m} \frac{f(y)}{|x|^{2}-|y|^{2}} d y \\
{\left[P^{-} f\right](x) } & :=\frac{1}{4 \pi^{2}} e^{i m \theta} \int_{\mathbb{R}^{2}} H_{m}^{(2)}(|x||\xi|) J_{m}(|\xi||y|) f(|y|) d \xi d y \\
& =\frac{1}{2} f(x)-\frac{i}{2 \pi^{2}} \int_{\mathbb{R}^{2}}\left|\frac{y}{x}\right|^{m} \frac{f(y)}{|x|^{2}-|y|^{2}} d y
\end{aligned}
$$

We denote the composition by $P_{N}^{ \pm}:=P^{ \pm} P_{N}$, and omit the equivariance class $m$ if it is clear. We record the following properties of $P^{ \pm}$.

Proposition 4.6 (Properties of $\left.P^{ \pm}([32,33,44])\right)$. The projection $P^{ \pm}$defined above satisfies the following properties:

(1) $P^{+}+P^{-}$acts as the identity on m-equivariant functions.

(2) For $|x| \gtrsim N^{-1}$ and $t \gtrsim N^{-2}$, the integral kernel obeys

$$
\left|\left[P_{N}^{ \pm} e^{\mp i t \Delta}\right](x, y)\right| \lesssim \begin{cases}(|x||y|)^{-\frac{1}{2}}|t|^{-\frac{1}{2}}, & |y|-|x| \sim N t, \\ \frac{N^{2}}{(N|x|)^{\frac{1}{2}}\langle N|y|\rangle^{\frac{1}{2}}}\left\langle N^{2} t+N|x|-N|y|\right\rangle^{-n} & \text { otherwise, }\end{cases}
$$

for all $n \geq 0$.

(3) For $|x| \gtrsim N^{-1}$ and $t \lesssim N^{-2}$, the integral kernel obeys

$$
\left|\left[P_{N}^{ \pm} e^{\mp i t \Delta}\right](x, y)\right| \lesssim \frac{N^{2}}{(N|x|)^{\frac{1}{2}}\langle N|y|\rangle^{\frac{1}{2}}}\langle N|x|-N|y|\rangle^{-n}
$$

for all $n \geq 0$.

(4) Fix $N>0$. Then

$$
\left\|\varphi_{\gtrsim \frac{1}{N}} P_{\geq N}^{ \pm} f\right\|_{L^{2}\left(\mathbb{R}^{2}\right)} \lesssim\|f\|_{L^{2}\left(\mathbb{R}^{2}\right)}
$$

with an $N$-independent constant.

Remark 4.7. These results are established in [32,33] for the radial in-out decomposition first, and then [44] proposes the $m$-equivariant case above, which is similar to the radial case due to the similar asymptotic behavior of Hankel functions and Bessel functions of all orders.

4.1.4. Almost periodic solution. A concentration compactness argument ensures that the threshold solution enjoys a certain strong concentration property in terms of charge.

\footnotetext{
${ }^{7}$ The computational detail can be found in [20, §6.521.2].
} 
This is characterized by the so-called almost periodicity modulo symmetries property; see $[44,53]$. The following theorem and lemma will be crucial to our proof.

Theorem 4.8 (Almost periodicity modulo symmetries). Let $\phi:[0, \infty) \times \mathbb{R}^{2} \rightarrow \mathbb{C}$ be a solution to (eCSS) that satisfies $\phi_{0} \in L_{m}^{2}$ for $m \geq 0,\left\|\phi_{0}\right\|_{L^{2}}=\|Q\|_{L^{2}}$ and

$$
\|\phi\|_{L_{t, x}^{4}\left([0, \infty) \times \mathbb{R}^{2}\right)}=\infty .
$$

Then $\phi$ is almost periodic modulo symmetries in the following sense: there exist functions $N:[0, \infty) \rightarrow \mathbb{R}^{+}$and $C: \mathbb{R}^{+} \rightarrow \mathbb{R}^{+}$, such that

$$
\int_{|x| \geq C(\eta) / N(t)}|\phi(t, x)|^{2} d x \leq \eta, \quad \int_{|\xi| \geq C(\eta) N(t)}|\hat{\phi}(t, \xi)|^{2} d \xi \leq \eta,
$$

for any $t \in[0, \infty)$ and $\eta>0$. Equivalently, the orbit $\left\{N(t)^{-1} \phi\left(t, \frac{x}{N(t)}\right): t \in[0, \infty)\right\}$ is precompact in $L_{x}^{2}\left(\mathbb{R}^{2}\right)$. We will refer to the functions $N$ and $C$ respectively as the frequency scale function and the compactness modulus function.

Moreover, $N(t)$ satisfies the local constancy property

$$
N(t) \sim_{\phi} N\left(t_{0}\right)
$$

whenever $t, t_{0} \in[0, \infty)$ and $\left|t-t_{0}\right| \lesssim_{\phi} N\left(t_{0}\right)^{-2}$, and

$$
N(t) \gtrsim_{\phi} N\left(t_{0}\right)\left\langle t-t_{0}\right\rangle^{-\frac{1}{2}}
$$

for all $t, t_{0} \in[0, \infty)$.

The first part of this theorem is not exactly the same as [44, Lemma 1.7], but, using the Palais-Smale condition modulo symmetries, is proved similarly to [53, Proposition 2.1]. The constancy property (4.4) is just the same as [32, Corollary 3.6]. The proof there works for general equations with a suitable standard Cauchy theory, which is provided in [44] for our case. Inequality (4.5) directly follows (4.4).

One important feature of an almost periodic solution is the following Duhamel formula, where the free evolution term disappears.

Lemma 4.9 (Nonscattering Duhamel). Let $\phi$ be an almost periodic solution to (eCSS) on $[0, \infty)$ in the sense of Theorem 4.8 . Then, for all $t \in[0, \infty)$,

$$
\phi(t)=-\lim _{T \rightarrow+\infty} i \int_{t}^{T} e^{i\left(t-t^{\prime}\right) \Delta} F\left(\phi\left(t^{\prime}\right)\right) d t^{\prime}
$$

as a weak limit in $L_{x}^{2}$, where $F(\phi)$ is the nonlinearity of (eCSS).

The proof in $[53, \S 6]$ for (NLS) merely depends on the linear evolution operator of the Schrödinger equation, and hence is still applicable in our (eCSS) setting.

\subsection{Reduction of the proof for Theorem 1.6}

In this subsection we will see how Theorem 1.6 reduces to the following frequency decay estimate. Therefore, the remaining task in the following subsections is just to prove this estimate. 
Proposition 4.10 (Frequency decay estimate). Let $m \geq 0, \phi_{0} \in H_{m}^{1}\left(\mathbb{R}^{2}\right),\left\|\phi_{0}\right\|_{L^{2}}=$ $\left\|\phi^{(m)}\right\|_{L^{2}}$ and $E[\phi]>0$. Let $\phi=u e^{i m \theta}$ be a solution to (eCSS) with initial data $\phi(0)=\phi_{0}$, and let $\phi$ blow up as $t \rightarrow+\infty$. Then there exists $\varepsilon>0$ such that for any dyadic number $N \geq 1$ we have

$$
\left\|\varphi_{>1} P_{N} \phi(t)\right\|_{L_{t}^{\infty} L_{x}^{2}\left([0, \infty) \times \mathbb{R}^{2}\right)} \lesssim\left\|\widetilde{P}_{N} \phi_{0}\right\|_{L_{x}^{2}}+N^{-1-\varepsilon} .
$$

This decay estimate will imply the localization of kinetic energy in Theorem 4.11 below. Then a contradiction argument using a truncated virial identity estimate will establish Theorem 1.6. To begin with, we state and prove Theorem 4.11.

Theorem 4.11 (Localization of kinetic energy). Let $m \geq 0$, and $\phi$ be as in Theorem 4.10. From Theorem 4.8, $\phi$ is almost periodic modulo symmetries. Let $N(t)$ be the corresponding scaling function; then there exists $\widetilde{C}: \mathbb{R}^{+} \rightarrow \mathbb{R}^{+}$such that for all $\eta>0$,

$$
\|\nabla \phi(t)\|_{L_{x}^{2}\left(\left\{|x|>\tilde{C}(\eta)\left\langle N(t)^{-1}\right\rangle\right\}\right)} \leq \eta .
$$

Proof. For any $\eta>0$ and $t \geq 0$, we will estimate

$$
\left\|\varphi_{>R} \nabla \phi(t)\right\|_{L^{2}}<\eta
$$

with $R=2 C\left(\eta_{1}\right)\left\langle N(t)^{-1}\right\rangle$, where $C$ is the compactness modulus function in Theorem 4.8 and $\eta_{1}$ is small enough to be determined.

First we add two frequency cutoffs, with $N_{0}$ to be determined:

$$
\left\|\varphi_{>R} \nabla \phi(t)\right\|_{2} \leq\left\|P_{\leq N_{0}} \varphi_{>R} \nabla \phi(t)\right\|_{2}+\left\|P_{>N_{0}} \varphi_{>R} \nabla \phi(t)\right\|_{2} .
$$

For the low-frequency part, we need to make the frequency truncation next to $\nabla$ and apply the charge concentration, through a mismatch estimate. Finally, we control the main term with localization of charge (4.3):

$$
\begin{aligned}
& \left\|P_{\leq N_{0}} \varphi_{>R} \nabla \phi(t)\right\|_{2} \\
& \lesssim \quad\left\|P_{\leq N_{0}} \varphi_{>R} \nabla P_{\leq 4 N_{0}} \varphi_{\leq \frac{R}{2}} \phi(t)\right\|_{2}+\left\|P_{\leq N_{0}} \varphi_{>R} \nabla P_{\leq 4 N_{0}} \varphi_{>\frac{R}{2}} \phi(t)\right\|_{2} \\
& \quad+\sum_{N>4 N_{0}}\left\|P_{\leq N_{0}} \varphi_{>R} \nabla P_{N} \phi(t)\right\|_{2} \\
& \quad{ }_{\phi} N_{0}^{-1} R^{-2}+N_{0}\left\|\varphi_{>\frac{R}{2}} \phi(t)\right\|_{2}+\sum_{N>4 N_{0}} N^{-1} R^{-2} \\
& \quad{ }_{\phi} N_{0}^{-1}+N_{0} \eta_{1} .
\end{aligned}
$$

For the high-frequency part, we use frequency decaying estimate Proposition 4.10 and almost orthogonality:

$$
\begin{gathered}
\left\|P_{>N_{0}} \varphi_{>R} \nabla \phi(t)\right\|_{2}^{2}=\sum_{N>N_{0}}\left\|P_{N} \varphi_{>R} \nabla \phi(t)\right\|_{2}^{2} \\
=\sum_{N>N_{0}}\left(\left\|P_{N} \varphi_{>R} P_{<\frac{N}{4}} \nabla \phi(t)\right\|_{2}^{2}+\left\|P_{N} \varphi_{>R} P_{>4 N} \nabla \phi(t)\right\|_{2}^{2}\right. \\
\left.+\left\|P_{N} \varphi_{>R} P_{\frac{N}{4} \leq \cdot \leq 4 N} \nabla \phi(t)\right\|_{2}^{2}\right)
\end{gathered}
$$




$$
\begin{aligned}
& \lesssim_{\phi} \sum_{N>N_{0}} N^{-2} R^{-4}+\sum_{N>\frac{1}{4} N_{0}}\left\|\varphi_{>R} \nabla P_{N} \phi(t)\right\|_{2}^{2} \\
& \lesssim_{\phi} N_{0}^{-2} R^{-4}+\sum_{N>\frac{N_{0}}{4}}\left(\left\|\varphi_{>R} \nabla \widetilde{P}_{N} \varphi_{\leq \frac{R}{2}} P_{N} \phi(t)\right\|_{2}^{2}+\left\|\varphi_{>R} \nabla \widetilde{P}_{N} \varphi_{>\frac{R}{2}} P_{N} \phi(t)\right\|_{2}^{2}\right) \\
& \lesssim_{\phi} N_{0}^{-2} R^{-4}+\sum_{N>\frac{N_{0}}{4}}\left[N^{-2} R^{-4}+N^{2}\left(N^{-2-2 \varepsilon}+\left\|P_{N} u(0)\right\|_{2}^{2}\right)\right] \\
& \lesssim_{\phi} N_{0}^{-2}+N_{0}^{-2 \varepsilon}+\left\|\nabla P_{>\frac{N_{0}}{4}} u(0)\right\|_{2}^{2} .
\end{aligned}
$$

Choosing $N_{0}$ large enough, and then $\eta_{1}$ small enough, the conclusion is proved.

Next, we are in place to prove our main theorem.

Proof of Theorem 1.6. Assume $E\left[\phi_{0}\right]>0$. Recall the truncated virial identity $V_{R}(t):=$ $\int_{\mathbb{R}^{2}} \chi_{R}|\phi|^{2}$ where $\chi_{R}$ is a cutoff for $|x|^{2}$ for $|x| \leq R$, defined in Corollary 2.5. Then we have (2.14) and a trivial bound

$$
\left|V_{R}(t)\right| \lesssim_{\phi} R^{2}
$$

Besides, since $\|\phi\|_{L^{2}}=\|Q\|_{L^{2}}$ and $\phi$ blows up as $t \rightarrow+\infty, \phi$ is an almost periodic solution according to Theorem 4.8, and we can apply Lemma 4.9 and Theorem 4.11. In the following argument, $N(t)$ and $C(\eta)$ are defined as in Theorem 4.8.

We distinguish two cases: either $N(t)$ is bounded from below (i.e. the charge, frequency and kinetic energy concentrate in a bounded area all the time) or converges to zero along a subsequence.

Case 1: $\inf _{t \geq 0} N(t)>0$. Let $\eta>0$ be a small constant chosen later. From localization of charge (Theorem 4.8) and kinetic energy (Theorem 4.11), there exists $R=R(\eta)=$ $2 C(\eta) /\left(\inf _{t} N(t)\right)$ such that

$$
\|\phi(t)\|_{L_{x}^{2}\left(|x| \geq \frac{R}{2}\right)}+\|\nabla \phi(t)\|_{L_{x}^{2}\left(|x| \geq \frac{R}{2}\right)} \leq 2 \eta
$$

for all $t \geq 0$. Then by the Gagliardo-Nirenberg inequality,

$$
\left\|\varphi_{\geq \frac{R}{2}} \phi(t)\right\|_{L_{x}^{4}} \lesssim_{\phi} \eta
$$

provided $R$ is chosen sufficiently large depending on $\eta$.

Hence, taking $\eta$ small enough depending on $E\left[\phi_{0}\right]$ and $R$ correspondingly large, the residual in (2.14) will be small enough. We obtain

$$
V_{R}^{\prime \prime}(t) \geq 8 E\left[\phi_{0}\right]>0
$$

for all $t \in[0, \infty)$, thus contradicting (4.8) for $t$ large enough.

Case 2: $\liminf _{t \rightarrow \infty} N(t)=0$. In this case we are not able to choose a fixed $R$ to guarantee uniform concentration. So the idea here is to consider the contradiction of the speed of divergence, due to the a priori bound (4.5). 
Choose the time sequence $t_{n} \nearrow \infty$ such that $N\left(t_{n}\right) \searrow 0$ and

$$
N\left(t_{n}\right)=\min _{0 \leq t \leq t_{n}} N(t)
$$

Again, let $\eta>0$ be a small constant and $R_{n}=2 C(\eta) / N\left(t_{n}\right)$; then

$$
\|\phi(t)\|_{L_{x}^{2}\left(|x| \geq \frac{R_{n}}{2}\right)}+\|\nabla \phi(t)\|_{L_{x}^{2}\left(|x| \geq \frac{R_{n}}{2}\right)}+\|\phi(t)\|_{L_{x}^{4 / d+2}\left(|x| \geq \frac{R_{n}}{2}\right)} \lesssim_{\phi} \eta
$$

for all $t \in\left[0, t_{n}\right]$. Thus, similarly, an $\eta$ small enough depending on $E\left[\phi_{0}\right]$ (but independent of $n$ ) implies

$$
V_{R_{n}}^{\prime \prime}(t) \geq 8 E\left[\phi_{0}\right]>0
$$

for all $t \in\left[0, t_{n}\right)$.

On the other hand, from the differential inequality for the truncated virial (3.21) and (4.8), we have

$$
\left|V_{R_{n}}^{\prime}(t)\right| \lesssim_{E\left(\phi_{0}\right)}\left(V_{R_{n}}(t)\right)^{1 / 2} \lesssim_{\phi} R_{n}
$$

for all $t \in\left[0, t_{n}\right]$. Thus, using the fundamental theorem of calculus and (4.13), we obtain

$$
E\left[\phi_{0}\right] t_{n} \leq\left|V_{R_{n}}^{\prime}(0)\right|+\left|V_{R_{n}}^{\prime}\left(t_{n}\right)\right| \lesssim_{\phi} R_{n} \lesssim_{\phi} N\left(t_{n}\right)^{-1} \lesssim_{\phi} t_{n}^{1 / 2} .
$$

Letting $n \rightarrow \infty$, we reach a contradiction since $t_{n} \rightarrow \infty$.

This finishes the proof of Theorem 1.6.

\subsection{Weak localization of kinetic energy}

Now the only goal for the rest of this paper is to prove Proposition 4.10. To achieve that, we need quantities controlling the solution uniformly for all time, and just the charge conservation is not enough in dimension 2. So we will prove the following uniform $\dot{H}^{1}$ boundedness property in this subsection, as preparation for proving Proposition 4.10.

Proposition 4.12 (Weak localization of kinetic energy). For $m \geq 0$, any $\phi \in H_{m}^{1},\|\phi\|_{L^{2}}=$ $\left\|\phi^{(m)}\right\|_{L^{2}}\left(\phi^{(m)}\right.$ is the m-equivariant soliton) and $E[\phi]>0$, then for all $c>0$,

$$
\left\|\varphi_{>c} \nabla \phi\right\|_{L^{2}} \lesssim_{c, E[\phi], m} 1
$$

Remark 4.13. Due to conservation of energy, this theorem indicates that $\nabla \phi(t)$ cannot be large in the region away from the origin. So it can also be regarded as a weaker localization of kinetic energy, compared with the strong one in Theorem 4.11.

To begin with, we need some spectral analysis of the linearized equation around the soliton.

4.3.1. Linearization of (eCSS) at the soliton. In this subsubsection we consider the linearization of (eCSS) at the $m$-equivariant soliton $\phi^{(m)}=e^{i m \theta} Q$, to see that the linearized operator can be written as a self-dual form

$$
\mathscr{L}_{Q}=L_{Q}^{*} L_{Q}
$$


Namely, if we consider the $u$-evolution formulation (1.12) for (eCSS), and denote $u=$ $Q+\varepsilon$, then (1.12) is equivalent to

$$
i \partial_{t} \varepsilon-L_{Q}^{*} L_{Q \varepsilon}=\text { (h.o.t.). }
$$

And then we record its spectral properties, the analysis of which largely depends on this self-dual structure. Most of these results appear in [35], so we omit their proof.

We begin with linearization of the Bogomol'nyi operator

$$
\begin{aligned}
\boldsymbol{D}_{+}^{(u)} & :=\partial_{r}-\frac{1}{r}\left(m+A_{\theta}[u]\right), \\
\boldsymbol{D}_{+}^{(u) *} & :=-\partial_{r}-\frac{1}{r}\left(1+m+A_{\theta}[u]\right) .
\end{aligned}
$$

Equation (1.12) can be written as a Hamiltonian equation,

$$
\partial_{t} u=-i \frac{\delta E}{\delta u}=-i \frac{\delta}{\delta u}\left(\frac{1}{2} \int\left|\boldsymbol{D}_{+}^{(u)} u\right|^{2}\right) .
$$

Corresponding to the quadrature structure of basic nonlinearity $A_{\theta}[u]$, we define the multiplication operator and its adjoint: ${ }^{8}$

$$
\begin{aligned}
& B_{f} g:=\frac{1}{r} \int_{0}^{r} \operatorname{Re}(\bar{f} g) r^{\prime} d r^{\prime}, \\
& B_{f}^{*} g=f \int_{r}^{\infty}(\operatorname{Re} g) d r^{\prime} .
\end{aligned}
$$

With that, we can represent those nonlinearities appearing in (eCSS):

$$
\begin{aligned}
& A_{\theta}[u] u=-\frac{1}{2} r\left(B_{u} u\right) u, \\
& A_{0}[u] u=-B_{u}^{*}\left[\frac{m}{r}|u|^{2}-\frac{1}{2}|u|^{2} B_{u} u\right] .
\end{aligned}
$$

Then assuming the decomposition at arbitrary profile $\omega$ is

$$
u=\omega+\varepsilon,
$$

we can further decompose the operator as

$$
\begin{gathered}
\boldsymbol{D}_{+}^{(u)}=\boldsymbol{D}_{+}^{(\omega)}+\left(B_{\omega} \varepsilon\right)+\frac{1}{2}\left(B_{\varepsilon} \varepsilon\right), \\
\boldsymbol{D}_{+}^{(u) *}=\boldsymbol{D}_{+}^{(\omega) *}+\left(B_{\omega} \varepsilon\right)+\frac{1}{2}\left(B_{\varepsilon} \varepsilon\right) .
\end{gathered}
$$

\footnotetext{
${ }^{8}$ We remark that the operator, as well as $A_{\theta}$, is only $\mathbb{R}$-linear, rather than $\mathbb{C}$-linear. So all the adjoints afterwards are viewed as being in the $\mathbb{R}$-Hilbert space $L^{2}\left(\mathbb{R}^{2} ; \mathbb{C}\right)$ equipped with the inner product $(u, v)_{r}=$ $\int \operatorname{Re}(u \bar{v})$.
} 
And hence

$$
\boldsymbol{D}_{+}^{(u)} u=\boldsymbol{D}_{+}^{(\omega)} u+L_{\omega} \varepsilon+N_{\omega}[\varepsilon]
$$

where the linear part $L_{\omega}$ and nonlinear part $N_{\omega}[\varepsilon]$ are given by

$$
\begin{aligned}
L_{\omega} & :=\boldsymbol{D}_{+}^{(\omega)}+\omega B_{\omega}, \\
N_{\omega}[\varepsilon] & :=\varepsilon B_{\omega} \varepsilon+\frac{1}{2} \omega B_{\varepsilon} \varepsilon+\frac{1}{2} \varepsilon B_{\varepsilon} \varepsilon,
\end{aligned}
$$

and the real adjoint of $L_{\omega}$ is

$$
L_{\omega}^{*} f=\boldsymbol{D}_{+}^{(\omega) *} f+B_{\omega}^{*}(\bar{\omega} f)
$$

In particular, when $\omega=Q$, using the self-dual relation $D_{+}^{(Q)} Q=0$, we have the following lemma:

Lemma 4.14 (Equation (eCSS) in self-dual form ([35])). Using the previous notation, the self-dual (eCSS) under the equivariant assumption is equivalent to

$$
i \partial_{t} u=L_{u}^{*} \boldsymbol{D}_{+}^{(u)} u
$$

Moreover, suppose $u=Q+\varepsilon$; it is equivalent to the linearized equation

$$
\begin{aligned}
i \partial_{t} \varepsilon-\mathscr{L}_{Q} \varepsilon & =L_{Q}^{*} N_{Q}[\varepsilon]+\left[\left(B_{Q} \varepsilon\right)+B_{Q}^{*}[\bar{\varepsilon} \cdot]+B_{\varepsilon}^{*}[Q \cdot]\right]\left[L_{Q} \varepsilon+N_{Q}[\varepsilon]\right] \\
& +\left[\frac{1}{2}\left(B_{\varepsilon} \varepsilon\right)+B_{\varepsilon}^{*}[\bar{\varepsilon} \cdot]\right]\left[L_{Q} \varepsilon+N_{Q}[\varepsilon]\right]
\end{aligned}
$$

where the linearized operator is

$$
\mathscr{L}_{Q}=L_{Q}^{*} L_{Q}
$$

Differentiating symmetries of (eCSS) at the static soliton $Q$, we obtain explicit algebraic identities satisfied by $\mathscr{L}_{Q}$. From phase and scaling symmetries we have

$$
\mathscr{L}_{Q}[i Q]=0, \quad \mathscr{L}_{Q}[\Lambda Q]=0,
$$

where $\Lambda$ is the generator for $L^{2}$-scaling,

$$
\Lambda f:=\left.\frac{d}{d \lambda}\right|_{\lambda=1} \lambda f(\lambda \cdot)=\left[1+r \partial_{r}\right] f
$$

One can indeed see that $i Q$ and $\Lambda Q$ span the kernel of $L_{Q}$. And the coercivity of $\mathscr{L}_{Q}$ follows from the factorization $\mathscr{L}_{Q}=L_{Q}^{*} L_{Q}$.

Lemma 4.15 (Kernel of $\left.L_{Q}([35])\right)$. If $f(r) e^{i m \theta}$ is a smooth m-equivariant function such that $L_{Q} f=0$, then $f \in \operatorname{span}_{\mathbb{R}}\{i Q, \Lambda Q\}$. 
Lemma 4.16 (Coercivity of $\left.\mathscr{L}_{Q}([35])\right)$. Let $m \geq 1$; we have ${ }^{9}$

$$
\begin{aligned}
\left\|L_{Q} u\right\|_{L^{2}} & \lesssim\|u\|_{\dot{H}_{m}^{1}} \quad \forall u e^{i m \theta} \in \dot{H}_{m}^{1}, \\
\left\|L_{Q} u\right\|_{L^{2}} & \gtrsim\|u\|_{\dot{H}_{m}^{1}} \quad \forall u e^{i m \theta} \in \dot{H}_{m}^{1},(u, i Q)_{r}=(u, \Lambda Q)_{r}=0 .
\end{aligned}
$$

And in the case $m=0,(4.22)$ and (4.23) are still true if we replace $\dot{H}_{m}^{1}$ by

$$
\|u\|_{\dot{\mathscr{H}}_{0}}^{2}:=\left\|\partial_{r} u\right\|_{L^{2}}^{2}+\left\|(1+r)^{-1} u\right\|_{L^{2}}^{2} .
$$

Remark 4.17. When $m \geq 1$ we have $i Q, \Lambda Q \in\left(\dot{H}_{m}^{1}\right)^{*}$, so the inner products in (4.23) are defined naturally, while for $m=0, i Q, \Lambda Q \notin\left(\dot{\mathscr{H}}_{0}\right)^{*}$ makes these inner products risky. Despite this, we use this lemma later only for $u \in H^{1}$ (see Lemma 4.19). Then it is not a problem since $Q \in L^{2}$ for all $m \geq 0$.

With all the information on $\mathscr{L}_{Q}$, we are able to compute the leading term in $E[Q+\cdot]$.

Lemma 4.18. For $m \geq 1, \varepsilon e^{i m \theta} \in H_{m}^{1}$ and $Q e^{i m \theta}$ the static soliton, we have

$$
\left|2 E\left[(Q+\varepsilon) e^{i m \theta}\right]-\left\|L_{Q} \varepsilon\right\|_{L^{2}}^{2}\right| \lesssim_{m}\|\varepsilon\|_{\dot{H}_{m}^{1}}^{2}\left(\sum_{k=1}^{4}\|\varepsilon\|_{H_{m}^{1}}^{k}\right) .
$$

And for $m=0$, (4.25) still holds if we replace $\dot{H}_{0}^{1}$ by $\dot{\mathscr{H}}_{0}$ defined in (4.24).

Proof of Lemma 4.18. Recall the decomposition (4.17) and note that $\boldsymbol{D}_{+}^{(Q)} Q=0$; we have

$$
\begin{aligned}
2 E\left[(Q+\varepsilon) e^{i m \theta}\right]-\left\|L_{Q \varepsilon}\right\|_{L^{2}}^{2} & =\int\left|\boldsymbol{D}_{+}^{(Q+\varepsilon)}(Q+\varepsilon)\right|^{2}-\left|L_{Q \varepsilon}\right|^{2} \\
& =\int\left|\boldsymbol{D}_{+}^{(Q)} Q+L_{Q^{\varepsilon}}+N_{Q}[\varepsilon]\right|^{2}-\left|L_{Q} \varepsilon\right|^{2} \\
& =\int\left|L_{Q^{\varepsilon}}+N_{Q}[\varepsilon]\right|^{2}-\left|L_{Q} \varepsilon\right|^{2} \\
& =\int 2 \operatorname{Re}\left(L_{Q} \varepsilon \cdot \overline{N_{Q}[\varepsilon]}\right)+\left|N_{Q}[\varepsilon]\right|^{2}
\end{aligned}
$$

Thus, (4.25) follows from the $L^{2}$ estimates for $L_{Q} \varepsilon$ and $N_{Q}[\varepsilon]$ (for $m=0$ again substitute $\dot{H}_{0}^{1}$ by $\left.\dot{\mathscr{H}}_{0}\right)$,

$$
\begin{aligned}
\left\|L_{Q} \varepsilon\right\|_{L^{2}} & \lesssim\|\varepsilon\|_{\dot{H}_{m}^{1}}, \\
\left\|N_{Q}[\varepsilon]\right\|_{L^{2}} & \lesssim\|\varepsilon\|_{\dot{H}_{m}^{1}}\left(\|\varepsilon\|_{H_{m}^{1}}+\|\varepsilon\|_{H_{m}^{1}}^{2}\right) .
\end{aligned}
$$

Inequality (4.26) comes from Lemma 4.16. Next we prove (4.27). Recall that

$$
N_{Q}[\varepsilon]=\varepsilon B_{Q} \varepsilon+\frac{1}{2} Q B_{\varepsilon} \varepsilon+\frac{1}{2} \varepsilon B_{\varepsilon} \varepsilon
$$

\footnotetext{
${ }^{9}$ Recall the notation $\|u\|_{\dot{H}_{m}^{1}}:=\left\|u e^{i m} \theta\right\|_{\dot{H}^{1}}$ from Section 2.1 .
} 
where

$$
B_{f} g=\frac{1}{r} \int_{0}^{r} \operatorname{Re}(\bar{f} g) r^{\prime} d r^{\prime}
$$

We distinguish two cases.

Case 1: $m \geq 1$. Now that $\frac{1}{r} \varepsilon$ is bounded in $L^{2}$, we have the estimate

$$
\left\|f_{1} B_{f_{2}} f_{3}\right\|_{L^{2}} \lesssim\left\|\frac{1}{r} f_{\alpha}\right\|_{L^{2}}\left\|f_{\beta}\right\|_{L^{2}}\left\|f_{\gamma}\right\|_{L^{2}} \quad \text { for }\{\alpha, \beta, \gamma\}=\{1,2,3\},
$$

by putting $\frac{1}{r}$ on $f_{1}$ or inside the integral of $B_{f_{2}} f_{3}$ as $\frac{1}{r^{\prime}}$ and then applying the CauchySchwarz inequality. Using this estimate and taking $\frac{1}{r}$ onto $\varepsilon$, (4.27) follows.

Case 2: $m=0$. In this case we need to be more careful. Recalling that $Q=\sqrt{8} \frac{1}{1+r^{2}}$, we see

$$
\left|B_{Q} \varepsilon(r)\right| \lesssim \frac{1}{r} \int_{0}^{r} \frac{1}{1+\left(r^{\prime}\right)^{2}}\left\|r^{\frac{1}{2}} \varepsilon\right\|_{L^{\infty}} \frac{1}{\left(r^{\prime}\right)^{\frac{1}{2}}} r^{\prime} d r^{\prime} \lesssim \min \left\{r^{\frac{1}{2}}, r^{-1}\right\}\left\|r^{\frac{1}{2}} \varepsilon\right\|_{L^{\infty}} .
$$

By the Strauss estimate in $\mathbb{R}^{2}$,

$$
|f(r)| \lesssim\|f\|_{\dot{H}^{1}(\{|x| \geq r\})}^{\frac{1}{2}}\|f\|_{L^{2}(\{|x| \geq r\})}^{\frac{1}{2}} r^{-\frac{1}{2}},
$$

we have

$$
\begin{aligned}
\left\|\varepsilon B_{Q} \varepsilon\right\|_{L^{2}} & \leq\left\|r^{\frac{1}{2}} \varepsilon\right\|_{L^{\infty}}\left\|r^{-\frac{1}{2}} B_{Q} \varepsilon\right\|_{L^{2}} \\
& \lesssim\left\|r^{\frac{1}{2}} \varepsilon\right\|_{L^{\infty}}^{2}\left\|\min \left\{1, r^{-\frac{3}{2}}\right\}\right\|_{L^{2}} \lesssim\|\varepsilon\|_{\dot{H}^{1}}\|\varepsilon\|_{L^{2}} .
\end{aligned}
$$

For $B_{\varepsilon} \varepsilon$

$$
\left|B_{\varepsilon} \varepsilon(r)\right| \lesssim \frac{1}{r} \int_{0}^{r} d r^{\prime}\left\|r^{\frac{1}{2}} \varepsilon\right\|_{L^{\infty}}^{2} \lesssim\left\|r^{\frac{1}{2}} \varepsilon\right\|_{L^{\infty}}^{2}
$$

then

$$
\begin{aligned}
\left\|Q B_{\varepsilon} \varepsilon\right\|_{L^{2}}+\left\|\varepsilon B_{\varepsilon} \varepsilon\right\|_{L^{2}} & \lesssim\left(\|Q\|_{L^{2}}+\|\varepsilon\|_{L^{2}}\right)\left\|r^{\frac{1}{2}} \varepsilon\right\|_{L^{\infty}}^{2} \\
& \lesssim\|\varepsilon\|_{\dot{H}^{1}}\left(\|\varepsilon\|_{L^{2}}+\|\varepsilon\|_{L^{2}}^{2}\right) .
\end{aligned}
$$

Thus (4.27) holds for $m \geq 0$.

\subsubsection{Modulation analysis.}

Lemma 4.19 (Rigidity of the ground state, quantitative version). Let $m \geq 0$ and $\phi^{(m)}=$ $Q e^{i m \theta}$ be the corresponding soliton. There exist constants $\eta>0, C>1, K>0$ such that the following is true.

Let $\phi=u e^{i m \theta} \in H_{m}^{1}$ be such that

$$
\|\phi\|_{L^{2}}=\left\|\phi^{(m)}\right\|_{L^{2}}, \quad\|\nabla \phi\|_{L^{2}}=\left\|\nabla \phi^{(m)}\right\|_{L^{2}}
$$

and

$$
E[\phi] \leq \eta
$$


Then there exist $\gamma_{0}=\gamma_{0}(\phi) \in \mathbb{R}, \lambda_{0}=\lambda_{0}(\phi)>0$ with

$$
\frac{1}{C} \leq \lambda_{0} \leq C
$$

such that

$$
\varepsilon=e^{i \gamma_{0}} \lambda_{0} u\left(\lambda_{0} \cdot\right)-Q
$$

satisfies the following:

(1) the orthogonality condition

$$
(\operatorname{Re}(\varepsilon), \Lambda Q)_{r}=(\operatorname{Im}(\varepsilon), Q)_{r}=0 ;
$$

(2) the bound of $\dot{H}_{m}^{1}$ norm (substituted by $\dot{\mathscr{H}}_{0}$ in (4.24) for $m=0$ case)

$$
\|\varepsilon\|_{\dot{H}_{m}^{1}} \leq K \sqrt{E[\phi]}
$$

Remark 4.20. Compared with the rigidity in Corollary 3.5, here we have a stronger quantitative estimate of the residual. But it is only an $\dot{H}_{m}^{1}$ control, which essentially comes from the $\dot{H}_{m}^{1}$ coercivity of $\mathscr{L}_{Q}$ (Lemma 4.16).

Proof of Lemma 4.19.

Step 1: Modulation method. We show that (4.31) holds. Define the $H^{1}$ neighborhood of $\phi^{(m)}$ as

$$
U_{\alpha}:=\left\{\phi \in H_{m}^{1}\left(\mathbb{R}^{2}\right):\left\|\phi-\phi^{(m)}\right\|_{H_{m}^{1}}<\alpha\right\} .
$$

For any $\gamma \in \mathbb{R}, \lambda>0, \phi=u e^{i m \theta} \in H_{m}^{1}$, define

$$
\varepsilon_{\lambda, \gamma}:=e^{i \gamma} \lambda u(\lambda \cdot)-Q
$$

We claim that there exists $\alpha_{0}>0$ and a unique map $(\lambda, \gamma): U_{\alpha_{0}} \rightarrow \mathbb{R}^{+} \times \mathbb{R}$ satisfying

$$
\left(\operatorname{Re}\left(\varepsilon_{\lambda, \gamma}\right), \Lambda Q\right)_{r}=\left(\operatorname{Im}\left(\varepsilon_{\lambda, \gamma}\right), Q\right)_{r}=0 .
$$

Furthermore, there exists a constant $K_{1}>0$ such that for $0<\alpha<\alpha_{0}, \phi \in U_{\alpha}$, we have

$$
\left\|\varepsilon_{\lambda, \gamma}\right\|_{H_{m}^{1}}+|\lambda-1|+|\gamma| \leq K_{1} \alpha .
$$

Consider the functional

$$
\rho_{1}(\phi, \lambda, \gamma):=\left(\operatorname{Re}\left(\varepsilon_{\lambda, \gamma}\right), \Lambda Q\right)_{r}, \quad \rho_{2}(\phi, \lambda, \gamma):=\left(\operatorname{Im}\left(\varepsilon_{\lambda, \gamma}\right), Q\right)_{r},
$$

and note that $\rho_{1}(Q, 1,0)=\rho_{2}(Q, 1,0)=0$; we only need to show that

$$
\left.\frac{\partial\left(\rho_{1}, \rho_{2}\right)}{\partial(\lambda, \gamma)}\right|_{(Q, 1,0)}
$$

is nondegenerate and then apply the implicit function theorem for a Banach space. Note that

$$
\left.\frac{\partial \varepsilon_{\lambda, \gamma}}{\partial \lambda}\right|_{(1,0)}=\Lambda u,\left.\quad \frac{\partial \varepsilon_{\lambda, \gamma}}{\partial \gamma}\right|_{(1,0)}=i u
$$


Thus

$$
(4.36)=\left(\begin{array}{cc}
\|\Lambda Q\|_{L^{2}}^{2} & 0 \\
0 & \|Q\|_{L^{2}}^{2}
\end{array}\right)
$$

is nondegenerate. So the claim holds, which implies that (4.31) holds if $\phi \in U_{\alpha_{0}}$.

Finally, using Corollary 3.5, for sufficiently small $\eta$, (4.29) implies $\phi \in U_{\alpha_{0}}$. We have finished the proof of (4.31). Also (4.30) comes immediately after (4.35).

Step 2: Quantitative control of the $\dot{H}_{m}^{1}$ norm. By Step 1, we already know $\varepsilon$ satisfies the orthogonality condition of Lemma 4.16, so we can use $\left\|L_{Q^{\varepsilon}}\right\|_{L^{2}}$ to control the $\dot{H}_{m}^{1}$ norm by (4.23). Recalling Lemma 4.18, this quantity is essentially $\sqrt{E(\phi)}$ with higher-order errors. Combined with the scaling property $\lambda_{0}^{2} E[\phi]=E\left[(Q+\varepsilon) e^{i m \theta}\right]$, for $m \geq 1$, we have

$$
\|\varepsilon\|_{\dot{H}_{m}^{1}}^{2} \leq C_{m}\left\|L_{Q} \varepsilon\right\|_{L^{2}}^{2} \leq 2 C_{m} \lambda_{0}^{2} E(\phi)+C_{m} C_{m}^{\prime}\|\varepsilon\|_{\dot{H}_{m}^{1}}^{2}\left(\sum_{k=1}^{4}\|\varepsilon\|_{H_{m}^{1}}^{k}\right) .
$$

From (4.35), if we take $\eta$ sufficiently small so that $\|\varepsilon\|_{H_{m}^{1}}$ is small enough and satisfies

$$
\left(\sum_{k=1}^{4}\|\varepsilon\|_{H_{m}^{1}}^{k}\right) C_{m} C_{m}^{\prime} \leq \frac{1}{2}
$$

then the left-hand side of (4.38) can absorb the last term on the right, which completes the proof for $m \geq 1$. As for the $m=0$ case, just replace the $\dot{H}_{0}^{1}$ norm by $\dot{\mathscr{H}}_{0}$ and the above estimates still hold.

4.3.3. Proof of Proposition 4.12. We can apply the modulation analysis to get the following nonsharp decomposition Proposition 4.21, which provides a useful bound for the distance to the soliton family.

Proposition 4.21 (Nonsharp decomposition of $H_{m}^{1}$ function with threshold charge). For $m \geq 0$, there exist $C_{1}, C_{2}>0$ such that for any $\phi=u e^{i m \theta} \in H_{m}^{1}$ with $\|\phi\|_{L^{2}}=\left\|\phi^{(m)}\right\|_{L^{2}}$ $\left(\phi^{(m)}\right.$ is the m-equivariant soliton), there exist $\theta_{0}=\theta_{0}(\phi) \in \mathbb{R}, \lambda=\lambda(\phi), \varepsilon e^{i m \theta}=$ $\varepsilon(\phi) e^{i m \theta} \in H_{m}^{1}$ for which we have

$$
u=\lambda e^{i \theta_{0}} Q(\lambda \cdot)+\varepsilon,
$$

where

$$
\frac{1}{C_{2}} \cdot \frac{\|\nabla \phi\|_{L^{2}}}{\left\|\nabla \phi^{(m)}\right\|_{L^{2}}} \leq \lambda \leq C_{2} \cdot \frac{\|\nabla \phi\|_{L^{2}}}{\left\|\nabla \phi^{(m)}\right\|_{L^{2}}} \quad \text { if }\|\nabla \phi\|_{L^{2}}^{2} \geq C_{1} E[\phi],
$$

and

$$
\lambda=1 \quad \text { if }\|\nabla \phi\|_{L^{2}}^{2} \leq C_{1} E[\phi] .
$$

The term $\varepsilon$ satisfies the bound

$$
\|\varepsilon\|_{\dot{H}_{m}^{1}} \lesssim \sqrt{E[\phi]}+1
$$


Proof of Proposition 4.21. Let $\phi=u e^{i m \theta} \in H_{m}^{1}$ and $\|\phi\|_{L^{2}}=\left\|\phi^{(m)}\right\|_{L^{2}}$. Rescale by $\mu=$ $\left\|\phi^{(m)}\right\|_{\dot{H}_{m}^{1}} /\|\phi\|_{\dot{H}_{m}^{1}}, \tilde{\phi}:=\mu \phi(\mu \cdot)$. Then

$$
\|\tilde{\phi}\|_{L}^{2}=\left\|\phi^{(m)}\right\|_{L^{2}}, \quad\|\tilde{\phi}\|_{\dot{H}_{m}^{1}}=\left\|\phi^{(m)}\right\|_{\dot{H}_{m}^{1}}, \quad E[\tilde{\phi}]=\frac{\left\|\phi^{(m)}\right\|_{\dot{H}_{m}^{1}}^{2}}{\|\phi\|_{\dot{H}_{m}^{1}}^{2}} E[\phi] .
$$

Take $C_{1}:=\left\|\phi^{(m)}\right\|_{\dot{H}_{m}^{1}}^{2} / \eta$ with $\eta$ as in Lemma 4.19. We distinguish two cases.

Case 1: $\|\phi\|_{\dot{H}_{m}^{1}}^{2} \geq C_{1} E[\phi]$. Note that the condition is exactly the smallness condition (4.29) for rigidity of $\tilde{\phi}$. We can apply Lemma 4.19 for $\tilde{\phi}$. Thus there exists $\tilde{\gamma}_{0} \in \mathbb{R}$ and $\tilde{\lambda}_{0} \in\left[\frac{1}{C}, C\right]$ and

Then after rescaling, we see that

$$
\begin{gathered}
\tilde{\varepsilon}:=e^{i \tilde{\gamma}_{0}} \tilde{\lambda}_{0} \tilde{u}\left(\tilde{\lambda}_{0} \cdot\right)-Q, \\
\|\tilde{\varepsilon}\|_{\dot{H}_{m}^{1}} \leq K \sqrt{E[\tilde{\phi}]} .
\end{gathered}
$$

$$
u=e^{i \theta_{0}} \lambda Q(\lambda \cdot)+\varepsilon,
$$

where

$$
\theta_{0}=-\tilde{\gamma}_{0}, \quad \lambda=\left(\tilde{\lambda}_{0} \mu\right)^{-1} \in\left[\frac{1}{C} \frac{\|\phi\|_{\dot{H}_{m}^{1}}}{\left\|\phi^{(m)}\right\|_{\dot{H}_{m}^{1}}}, C \frac{\|\phi\|_{\dot{H}_{m}^{1}}}{\left\|\phi^{(m)}\right\|_{\dot{H}_{m}^{1}}}\right], \quad \varepsilon=\lambda \tilde{\varepsilon}(\lambda \cdot) .
$$

Since $\lambda \sim \mu^{-1}$, by rescaling of (4.40) we immediately obtain (4.39).

Case 2: $\|\phi\|_{\dot{H}_{m}^{1}}^{2} \leq C_{1} E[\phi]$. We just set $\theta_{0}=0, \lambda=1$ and $\varepsilon=u-Q$. Then (4.39) comes from a rough bound

$$
\|\varepsilon\|_{\dot{H}_{m}^{1}} \leq\|Q\|_{\dot{H}_{m}^{1}}+\|u\|_{\dot{H}_{m}^{1}} \leq\|Q\|_{\dot{H}_{m}^{1}}+\|\phi\|_{\dot{H}_{m}^{1}} \lesssim_{m} 1+\sqrt{E[\phi]} .
$$

Finally, we conclude the proof of Proposition 4.12:

Proof of Proposition 4.12. Apply Proposition 4.21. Then, when $\|\nabla \phi\|_{L^{2}}^{2} \leq C_{1} E[\phi]$, the bound is obvious.

If $\|\nabla \phi\|_{L^{2}}^{2} \geq C_{1} E[\phi]$,

$$
\begin{aligned}
\left\|\varphi_{>c} \nabla \phi\right\|_{L^{2}} & \lesssim\left\|\varphi_{>c} \partial_{r} \phi\right\|_{L^{2}}+\left\|\varphi_{>c} \frac{1}{r} \phi\right\|_{L^{2}} \\
& \lesssim\left\|\varphi_{>c} \partial_{r}(\lambda Q(\lambda \cdot))\right\|_{L^{2}}+\left\|\varphi_{>c} \partial_{r} \varepsilon\right\|_{L^{2}}+\|\phi\|_{L^{2}}
\end{aligned}
$$

The third term is bound by the threshold charge $\|\phi\|_{L^{2}}=\|Q\|_{L^{2}}$, and the second by (4.39). For the first term, recalling that $Q=C_{m} \frac{r^{m}}{1+r^{2(m+1)}}$ has a good decay property away from the origin,

$$
\begin{aligned}
\|\lambda Q(\lambda \cdot)\|_{\dot{H}_{m}^{1}(|x| \geq c)}^{2} & \lesssim_{m} \int_{c}^{\infty} \lambda^{2}\left(\lambda^{2}\left|\partial_{r} Q(\lambda r)\right|^{2}+\frac{1}{r^{2}} Q(\lambda r)^{2}\right) r d r \\
& \lesssim_{m} \int_{c}^{\infty} \lambda^{4}\left(\frac{1}{\lambda r}\right)^{2 m+6} r d r=\lambda^{2} \int_{\lambda c}^{\infty} s^{-2 m-5} d r \\
& \lesssim_{m} \lambda^{-2 m-2} c^{-2 m-4} \lesssim_{m, E[\phi], c} 1 .
\end{aligned}
$$

The last inequality follows from $\lambda \sim_{m}\|\phi\|_{\dot{H}_{m}^{1}} \gtrsim E[\phi]>0$ in this case. 


\subsection{Proof of Proposition 4.10}

To establish this proposition, we first summarize the estimate we will use for the nonlinearity.

Lemma 4.22 (Estimate for nonlinearity). Let $\phi$ be as in Proposition 4.10 and $F(\phi)$ be the nonlinearity (1.14) of (eCSS); we have the following estimates:

$$
\begin{aligned}
\left\|\varphi_{>\frac{1}{4}} F(\phi)\right\|_{L_{t}^{\infty} L_{x}^{2}\left([0, \infty) \times \mathbb{R}^{2}\right)} & \lesssim_{\phi} 1, \\
\left\|\varphi_{>\frac{1}{4}} \partial_{r}(F(\phi))\right\|_{L_{t}^{\infty} L_{x}^{2}\left([0, \infty) \times \mathbb{R}^{2}\right)} & \lesssim_{\phi} 1, \\
\left\|\varphi_{>} F(\phi)\right\|_{L_{t}^{\infty} L_{x}^{1}\left([0, \infty) \times \mathbb{R}^{2}\right)} & \lesssim_{\phi} T^{-\frac{1}{2}}, \\
\left\|\varphi_{>} \partial_{r}(F(\phi))\right\|_{L_{t}^{\infty} L_{x}^{1}\left([0, \infty) \times \mathbb{R}^{2}\right)} & \lesssim_{\phi} T^{-\frac{1}{2}} .
\end{aligned}
$$

Next we prove Proposition 4.10 with these estimates, and defer their proof till the end of this subsection.

Proof of Proposition 4.10. We begin by projecting $\phi$ onto incoming and outgoing waves, and then decompose them with Lemma 4.9 (since $\phi$ is almost periodic from Theorem 4.8) and the standard Duhamel formula respectively:

$$
\begin{aligned}
\varphi_{>1} P_{N} \phi(t)= & \varphi_{>1} P_{N}^{+} \phi(t)+\varphi_{>1} P_{N}^{-} \phi(t) \\
= & \varphi_{>1} P_{N}^{-} e^{i t \Delta} \phi_{0} \\
& +i \int_{0}^{\infty} \varphi_{>1} P_{N}^{+} e^{-i \tau \Delta} F(\phi(t+\tau)) d \tau \\
& -i \int_{0}^{t} \varphi_{>1} P_{N}^{-} e^{i \tau \Delta} F(\phi(t-\tau)) d \tau .
\end{aligned}
$$

The last two integrals should be understood in the weak $L_{x}^{2}$ sense, for which our estimate is still valid thanks to Fatou's property. Since $\varphi_{>1} P_{N}^{-}$is a bounded operator from $L^{2}$ to $L^{2}$ by Proposition 4.6 (4), the first term is controlled by the Strichartz estimate

$$
\|(4.45)\|_{L_{x}^{2}} \lesssim\left\|\widetilde{P}_{N} \phi_{0}\right\|_{L^{2}}
$$

Here, we only give the details of the estimate of $(4.46) ;(4.47)$ is done in a similar way and thus is omitted. We start by decomposing: ${ }^{10}$

$$
\begin{aligned}
i \varphi_{>1} \int_{0}^{\infty} P_{N}^{+} e^{-i \tau \Delta} F(\phi(t+\tau)) d \tau \\
=i \varphi_{>1} \int_{0}^{N^{-1}} P_{N}^{+} e^{-i \tau \Delta} \varphi_{\leq \frac{1}{2}} F(\phi(t+\tau)) d \tau
\end{aligned}
$$

\footnotetext{
${ }^{10}$ We remark that the partitioning point $\frac{N \tau}{2}$ in (4.50) and (4.51) may be modified to $\frac{N \tau}{C}$ with $C \ll 1$, in order that the stationary phase in Proposition 4.6(2) will not be touched. Here we take $C=2$ for simplicity.
} 


$$
\begin{aligned}
& +i \varphi_{>1} \int_{0}^{N^{-1}} P_{N}^{+} e^{-i \tau \Delta} \varphi_{>\frac{1}{2}} F(\phi(t+\tau)) d \tau \\
& +i \varphi_{>1} \int_{N^{-1}}^{\infty} P_{N}^{+} e^{-i \tau \Delta} \varphi_{\leq \frac{N \tau}{2}} F(\phi(t+\tau)) d \tau \\
& +i \varphi_{>1} \int_{N^{-1}}^{\infty} P_{N}^{+} e^{-i \tau \Delta} \varphi_{>\frac{N \tau}{2}} F(\phi(t+\tau)) d \tau
\end{aligned}
$$

The remaining part of this proof is devoted to estimating these four parts.

Estimate of (4.48). This is the tail term considering the decaying estimate of $P_{N}^{+} e^{-i t \Delta}$ as in Proposition 4.6. To make use of the $\dot{H}^{1}$ control away from the origin, we use the equation $\left(i \partial_{t}+\Delta\right) \phi=F(\phi)$ to replace $F(\phi)$ by $\left(i \partial_{t}+\Delta\right) \phi$. Note that

$$
\begin{aligned}
(4.48)= & i \varphi_{>1} P_{N}^{+} \int_{0}^{\frac{1}{N}} e^{-i \tau \Delta}\left(\varphi_{\leq \frac{1}{2}}\left(i \partial_{\tau}+\Delta\right) \phi(t+\tau)\right) d \tau \\
= & -\varphi_{>1} P_{N}^{+} e^{-i \frac{1}{N} \Delta}\left(\varphi_{\leq \frac{1}{2}} \phi\left(t+\frac{1}{N}\right)\right) \\
& +\varphi_{>1} P_{N}^{+}\left(\varphi_{\leq \frac{1}{2}} \phi(t)\right) \\
& -i \varphi_{>1} P_{N}^{+} \int_{0}^{\frac{1}{N}} e^{-i \tau \Delta} \phi(t+\tau) \Delta \varphi_{\leq \frac{1}{2}} d \tau \\
& -2 i \varphi_{>1} P_{N}^{+} \int_{0}^{\frac{1}{N}} e^{-i \tau \Delta} \nabla \phi(t+\tau) \cdot \nabla \varphi_{\leq \frac{1}{2}} d \tau,
\end{aligned}
$$

where the second equality used the commutator $\left[\partial_{\tau}, e^{-i \tau \Delta}\right]=-i \Delta$ and integration by parts on $\tau$. These four terms are going to be estimated in the same manner, so we only estimate (4.55) as an example. By Proposition 4.6, the kernel obeys the estimate

$$
\begin{aligned}
\left|\left[\varphi_{>1} P_{N}^{+} e^{-i \tau \Delta} \kappa_{\leq \frac{1}{2}}\right](x, y)\right| & \lesssim N^{2}\langle N|x|-N|y|\rangle^{-n} \kappa_{|x|>1} \kappa_{|y| \leq \frac{1}{2}} \\
& \lesssim N^{2-n / 2}\langle x-y\rangle^{-n / 2}, \quad \tau \in\left[0, \frac{1}{N^{2}}\right], \\
\left|\left[\varphi_{>1} P_{N}^{+} e^{-i \tau \Delta} \kappa_{\leq \frac{1}{2}}\right](x, y)\right| & \lesssim N^{2}\left\langle N^{2} \tau+N|x|-N|y|\right\rangle^{-n} \kappa_{|x|>1} \kappa_{|y| \leq \frac{1}{2}} \\
& \lesssim N^{2}\left\langle N^{2} \tau+N|x|+N|y|\right\rangle^{-n} \kappa_{|x|>1} \kappa_{|y| \leq \frac{1}{2}} \\
& \lesssim N^{2-n / 2}\langle x-y\rangle^{-n / 2}, \quad \tau \in\left[\frac{1}{N^{2}}, \frac{1}{N}\right],
\end{aligned}
$$

for any $n>0$, where $\kappa_{\leq \frac{1}{2}}$ is a characteristic function. So Young's inequality tells us

$$
\begin{aligned}
\|(4.55)\|_{L_{x}^{2}} & =2\left\|\int_{0}^{\frac{1}{N}} \varphi_{>1} P_{N}^{+} e^{-i \tau \Delta} \kappa_{\leq \frac{1}{2}} \nabla \phi(t+\tau) \cdot \nabla \varphi_{\leq \frac{1}{2}} d \tau\right\|_{L_{x}^{2}} \\
& \lesssim \frac{1}{N}\left\|\int\left[\varphi_{>1} P_{N}^{+} e^{-i \tau \Delta} \kappa_{\leq \frac{1}{2}}\right](x, y)\left(\nabla \phi(t+\tau) \cdot \nabla \varphi_{\leq \frac{1}{2}}\right)(y) d y\right\|_{L_{\tau}^{\infty} L_{x}^{2}\left(\left[0, \frac{1}{N}\right] \times \mathbb{R}^{2}\right)} \\
& \lesssim N^{-10}\left\|\nabla \phi(t+\tau) \cdot \nabla \varphi_{\leq \frac{1}{2}}\right\|_{L_{\tau}^{\infty} L_{x}^{2}\left(\left[0, \frac{1}{N}\right] \times \mathbb{R}^{2}\right)} \\
& \lesssim N^{-10}\left\|\varphi_{>\frac{1}{4}} \partial_{r} \phi\right\|_{L_{t}^{\infty} L_{x}^{2}\left([0, \infty) \times \mathbb{R}^{2}\right)} \lesssim_{\phi} 1 .
\end{aligned}
$$


The other terms have similar estimates, so overall,

$$
\|(4.48)\|_{L_{x}^{2}} \lesssim N^{-9}
$$

Estimate of (4.49). We use nonlinearity estimates (4.41) and (4.42) here:

$$
\begin{aligned}
\|(4.49)\|_{L_{x}^{2}} & \lesssim\left\|\widetilde{P}_{N} \varphi_{>\frac{1}{2}} F(\phi)\right\|_{L_{\tau}^{1} L_{x}^{2}\left(\left[t, t+\frac{1}{N}\right] \times \mathbb{R}^{2}\right)} \\
& \lesssim \frac{1}{N}\left\|\widetilde{P}_{N} \varphi_{>\frac{1}{2}} F(\phi)\right\|_{L_{\tau}^{\infty} L_{x}^{2}\left([0, \infty) \times \mathbb{R}^{2}\right)} \\
& \lesssim \frac{1}{N^{2}}\left\|\widetilde{P}_{N}|\nabla|\left(\varphi_{>\frac{1}{2}} F(\phi)\right)\right\|_{L_{\tau}^{\infty} L_{x}^{2}\left([0, \infty) \times \mathbb{R}^{2}\right)} \\
& \lesssim \frac{1}{N^{2}}\left[\left\|\varphi_{>\frac{1}{4}} F(\phi)\right\|_{L_{\tau}^{\infty} L_{x}^{2}\left([0, \infty) \times \mathbb{R}^{2}\right)}+\left\|\varphi_{>\frac{1}{4}} \frac{1}{r} F(\phi)\right\|_{L_{\tau}^{\infty} L_{x}^{2}\left([0, \infty) \times \mathbb{R}^{2}\right)}\right. \\
& \left.\quad+\left\|\varphi_{>\frac{1}{4}} \partial_{r} F(\phi)\right\|_{\left.L_{\tau}^{\infty} L_{x}^{2}\left([0, \infty) \times \mathbb{R}^{2}\right)\right]}\right] \\
& \lesssim \frac{1}{N^{2}} .
\end{aligned}
$$

Estimate of (4.50). This is still a tail term, so we estimate it similarly to (4.48). Since

$$
\begin{aligned}
\|(4.50)\|_{L_{x}^{2}} & \leq \limsup _{T \rightarrow+\infty}\left\|i \varphi_{>1} \int_{N^{-1}}^{T} P_{N}^{+} e^{-i \tau \Delta} \varphi_{\leq \frac{N \tau}{2}} F(\phi(t+\tau)) d \tau\right\|_{L_{x}^{2}} \\
& :=\limsup _{T \rightarrow+\infty}\left\|(4.50)_{T}\right\|_{L_{x}^{2}},
\end{aligned}
$$

we aim to derive a uniform estimate of $(4.50)_{T}$. Using the equation, we have

$$
\begin{aligned}
(4.50)_{T}= & i \varphi_{>1} P_{N}^{+} \int_{\frac{1}{N}}^{T} e^{-i \tau \Delta}\left(\varphi_{\leq \frac{N \tau}{2}}\left(i \partial_{\tau}+\Delta\right) \phi(t+\tau)\right) d \tau \\
= & -\varphi_{>1} P_{N}^{+} e^{-i T \Delta}\left(\varphi_{\leq \frac{N T}{2}} \phi(t+T)\right) \\
& +\varphi_{>1} P_{N}^{+} e^{-i \frac{1}{N} \Delta}\left(\varphi_{\leq \frac{1}{2}} \phi\left(t+\frac{1}{N}\right)\right) \\
& -i \varphi_{>1} P_{N}^{+} \int_{\frac{1}{N}}^{T} e^{-i \tau \Delta} \phi(t+\tau) \Delta \varphi_{\leq \frac{N \tau}{2}} d \tau \\
& -2 i \varphi_{>1} P_{N}^{+} \int_{\frac{1}{N}}^{T} e^{-i \tau \Delta} \nabla \phi(t+\tau) \cdot \nabla \varphi_{\leq \frac{N \tau}{2}} d \tau \\
& -\varphi_{>1} P_{N}^{+} \int_{\frac{1}{N}}^{T} e^{-i \tau \Delta}\left(\frac{r}{N \tau^{2}} \partial_{r} \varphi_{\leq \frac{1}{2}}\left(\frac{r}{N \tau}\right) \phi(t+\tau)\right) d \tau .
\end{aligned}
$$

Again we have a decay estimate of the kernel for $\tau \geq \frac{1}{N}$ :

$$
\begin{aligned}
\left|\left[\varphi_{>1} P_{N}^{+} e^{-i \tau \Delta} \kappa_{\leq \frac{N \tau}{2}}\right](x, y)\right| & \lesssim N^{d}\left\langle N^{2} \tau+N|x|-N|y|\right\rangle^{-n} \kappa_{|x|>1} \kappa_{|y| \leq \frac{N \tau}{2}} \\
& \lesssim N^{2}\left\langle N^{2} \tau+N|x|+N|y|\right\rangle^{-n} \kappa_{|x|>1} \kappa_{|y| \leq \frac{N \tau}{2}} \\
& \lesssim N^{2-n}(N \tau)^{-n / 2}\langle x-y\rangle^{-n / 2} .
\end{aligned}
$$


Then we can estimate (4.56) as an example of those terms from $(4.50)_{T}$ :

$$
\begin{aligned}
\|(4.56)\|_{L_{x}^{2}} & =\left\|\int_{\frac{1}{N}}^{T} \int_{\mathbb{R}^{2}} \varphi_{>1} P_{N}^{+} e^{-i \tau \Delta} \kappa_{\leq \frac{N \tau}{2}}(x, y)\left(\frac{|y|}{N \tau^{2}} \partial_{r} \varphi_{\leq \frac{1}{2}}\left(\frac{|y|}{N \tau}\right) \phi(t+\tau, y)\right) d y d \tau\right\|_{L_{x}^{2}} \\
& \lesssim N^{-10} \int_{\frac{1}{N}}^{T}(N \tau)^{-6}\left\|\left(\frac{|y|}{N \tau^{2}} \partial_{r} \varphi_{\leq \frac{1}{2}}\left(\frac{|y|}{N \tau}\right) \phi(t+\tau, y)\right)\right\|_{L_{x}^{2}\left([0, \infty) \times \mathbb{R}^{2}\right)} d \tau \\
& \lesssim N^{-10} \int_{\frac{1}{N}}^{T}(N \tau)^{-6} \tau^{-1}\|\phi\|_{L_{t}^{\infty} L_{x}^{2}\left([0, \infty) \times \mathbb{R}^{2}\right)} d \tau \\
& \lesssim N^{-9} .
\end{aligned}
$$

To conclude, we have

$$
\|(4.50)\|_{L_{x}^{2}} \leq \limsup _{T \rightarrow+\infty}\left\|(4.50)_{T}\right\|_{L_{x}^{2}} \lesssim N^{-9}
$$

Estimate of (4.51). We first further decompose (4.51) by using $\varphi_{>\frac{N \tau}{2}}=\varphi_{>\frac{N \tau}{4}} \varphi_{>\frac{N \tau}{2}}$ and then introducing a frequency projection:

$$
\begin{aligned}
(4.51)= & i \varphi_{>1} \int_{N^{-1}}^{\infty} P_{N}^{+} e^{-i \tau \Delta} \varphi_{>\frac{N \tau}{4}} P_{>\frac{N}{8}} \varphi_{>\frac{N \tau}{2}} F(\phi(t+\tau)) d \tau \\
& +i \varphi_{>1} \int_{N^{-1}}^{\infty} P_{N}^{+} e^{-i \tau \Delta} \varphi_{>\frac{N \tau}{4}} P_{\leq \frac{N}{8}} \varphi_{>\frac{N \tau}{2}} F(\phi(t+\tau)) d \tau .
\end{aligned}
$$

Expression (4.57) can be estimated by a weighted Strichartz estimate (Lemma 4.5), and estimates of nonlinearity (4.43) and (4.44):

$$
\begin{aligned}
& \|(4.57)\|_{L_{x}^{2}} \lesssim\left\|\int_{\frac{1}{N}}^{\infty} e^{-i \tau \Delta} \varphi_{>\frac{N \tau}{4}} P_{>\frac{N}{8}} \varphi_{>\frac{N \tau}{2}} F(\phi(t+\tau)) d \tau\right\|_{L_{x}^{2}} \\
& \lesssim\left\||x|^{-\frac{1}{2}} \varphi_{>\frac{N \tau}{4}} P_{>\frac{N}{8}} \varphi_{>\frac{N \tau}{2}} F(\phi(t+\tau))\right\|_{L_{\tau}^{\frac{4}{3}} L_{x}^{1}\left(\left[\frac{1}{N}, \infty\right) \times \mathbb{R}^{2}\right)} \\
& \lesssim\left\|(N \tau)^{-\frac{1}{2}}\right\| P_{>\frac{N}{8}} \varphi_{>\frac{N \tau}{2}} F(\phi(t+\tau))\left\|_{L_{x}^{1}}\right\|_{L_{\tau}^{\frac{4}{3}}\left(\left[\frac{1}{N}, \infty\right)\right)} \\
& \lesssim N^{-\frac{3}{2}}\left\|\tau^{-\frac{1}{2}}\right\| P_{>\frac{N}{8}}|\nabla| \varphi_{>\frac{N \tau}{2}} F(\phi(t+\tau))\left\|_{L_{x}^{1}}\right\|_{L_{\tau}^{\frac{4}{3}}\left(\left[\frac{1}{N}, \infty\right)\right)} \\
& \lesssim N^{-\frac{3}{2}} \| \tau^{-\frac{1}{2}}\left[\left\|\varphi_{>\frac{N \tau}{4}}(N \tau)^{-1} F(\phi(t+\tau))\right\|_{L_{x}^{1}}+\left\|\varphi_{>\frac{N \tau}{4}} \frac{1}{r} F(\phi(t+\tau))\right\|_{L_{x}^{1}}\right. \\
& \left.+\left\|\varphi_{>\frac{N \tau}{4}} \partial_{r}(F(\phi(t+\tau)))\right\|_{L_{x}^{1}}\right] \|_{L_{\tau}^{\frac{4}{3}}\left(\left[\frac{1}{N}, \infty\right)\right)} \\
& \lesssim N^{-\frac{3}{2}}\left\|\tau^{-\frac{1}{2}}(N \tau)^{-\frac{1}{2}}\right\|_{L_{\tau}^{\frac{4}{3}}\left(\left[\frac{1}{N}, \infty\right)\right)} \\
& \lesssim N^{-\frac{7}{4}} \text {. }
\end{aligned}
$$


And (4.58) easily follows from mismatch estimate Lemma 4.3 and (4.41):

$$
\begin{aligned}
\|(4.58)\|_{L_{x}^{2}} & \lesssim\left\|\widetilde{P}_{N} \varphi_{>\frac{N \tau}{4}} P_{\leq \frac{N}{8}} \varphi_{>\frac{N \tau}{2}} F(\phi(t+\tau))\right\|_{L_{\tau}^{1} L_{x}^{2}\left(\left[\frac{1}{N}, \infty\right) \times \mathbb{R}^{2}\right.} \\
& \lesssim N^{-10}\left\|(N \tau)^{-10}\right\|_{L_{\tau}^{1}\left(\left[\frac{1}{N}, \infty\right)\right)}\left\|\varphi_{>\frac{1}{4}} F(\phi)\right\|_{L_{t}^{\infty} L_{x}^{2}\left([0, \infty) \times \mathbb{R}^{2}\right)} \\
& \lesssim N^{-11} .
\end{aligned}
$$

Thus we have proved

$$
\|(4.51)\|_{L_{x}^{2}} \lesssim N^{-\frac{7}{4}}
$$

Collecting estimates for (4.48)-(4.51), we have shown $\|(4.46)\|_{L_{x}^{2}} \lesssim N^{-1-\frac{3}{4}}$. A similar estimate holds for (4.47), and thus we have completed the proof of Proposition 4.10.

Finally, let us complete the proof of the nonlinear estimates, based on charge conservation and the weak localization of kinetic energy in Proposition 4.12.

Proof of Lemma 4.22. Note that $F(\phi)=F(u) e^{i m \theta}$. For simplicity, we replace $F(\phi)$ by $F(u)$ as the target estimates will remain the same. Define

$$
A_{0}^{(1)} u:=-u \int_{r}^{\infty} \frac{A_{\theta}}{s^{2}}|u|^{2} s d s, \quad A_{0}^{(2)} u:=-u \int_{r}^{\infty} \frac{m}{s^{2}}|u|^{2} s d s
$$

so that $A_{0}=A_{0}^{(1)}+A_{0}^{(2)}$, and then define

$$
\begin{gathered}
N_{1}(u)=-|u|^{2} u, \quad N_{2}(u)=\frac{2 m}{r^{2}} A_{\theta} u, \quad N_{3}(u)=\frac{A_{\theta}^{2}}{r^{2}} u, \\
N_{4}(u):=A_{0}^{(1)} u, \quad N_{5}(u):=A_{0}^{(2)} u .
\end{gathered}
$$

Thus $F(u)=\sum_{i=1}^{5} N_{i}(u)$. We will deal with each $N_{i}(u)$ separately.

Under the assumption of Proposition 4.10, we know $\phi(t)$ satisfies Proposition 4.12 and hence

$$
\left\|\phi_{>\frac{1}{16}} \nabla \phi\right\|_{L_{t}^{\infty} L_{x}^{2}\left([0, \infty) \times \mathbb{R}^{2}\right)} \lesssim_{\phi} 1 .
$$

And hence by the Strauss estimate (4.28), for $r \geq \frac{1}{16}$,

$$
\begin{aligned}
\|u(t, r)\|_{L_{t}^{\infty}([0, \infty))} & \leq\left\|\partial_{r} u(t)\right\|_{L_{t}^{\infty} L_{x}^{2}\left([0, \infty) \times\left\{|x| \geq \frac{1}{16}\right\}\right)}^{\frac{1}{2}}\|u\|_{L_{t}^{\infty} L_{x}^{2}\left([0, \infty) \times\left\{|x| \geq \frac{1}{16}\right\}\right)}^{\frac{1}{2}} r^{-\frac{1}{2}} \\
& \lesssim u r^{-\frac{1}{2}}
\end{aligned}
$$

In particular,

$$
\left\|\varphi_{>\frac{1}{8}} u\right\|_{L_{t}^{\infty} L_{x}^{\infty}\left([0, \infty) \times \mathbb{R}^{2}\right)} \lesssim u 1 .
$$

In the following estimate, our tools will be (4.60) (4.61) and conservation of charge

$$
\|u\|_{L_{t}^{\infty} L_{x}^{2}\left([0, \infty) \times \mathbb{R}^{2}\right)} \lesssim u 1 .
$$

We begin with estimates of $A_{\theta}, A_{0}$ :

$$
\left\|A_{\theta}\right\|_{L_{t}^{\infty} L_{x}^{\infty}} \lesssim\|u\|_{L_{t}^{\infty} L_{x}^{2}}^{2} \lesssim u 1,
$$




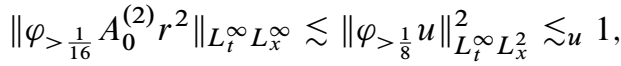

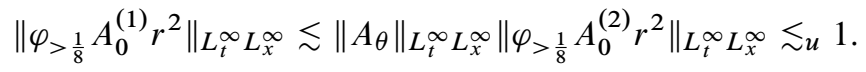

Now (4.41) and (4.42) easily follow from these bounds. We show estimates of $N_{1}$ and $N_{2}$ as examples. The following estimates are valid uniformly in time:

$$
\begin{aligned}
& \left\|\varphi_{>\frac{1}{4}} N_{1}(u)\right\|_{L_{x}^{2}} \leq\left\|\varphi_{>\frac{1}{8}} u\right\|_{L_{x}^{2}}\left\|\varphi_{>\frac{1}{8}} u\right\|_{L_{x}^{\infty}}^{2} \lesssim u 1, \\
& \left\|\varphi_{>\frac{1}{4}} \partial_{r}\left(N_{1}(u)\right)\right\|_{L_{x}^{2}} \lesssim\left\|\varphi_{>\frac{1}{8}} \partial_{r} u\right\|_{L_{x}^{2}}\left\|\varphi_{>\frac{1}{8}} u\right\|_{L_{x}^{\infty}}^{2} \lesssim u 1, \\
& \left\|\varphi_{>\frac{1}{4}} N_{2}(u)\right\|_{L_{x}^{2}} \leq\left\|\varphi_{>\frac{1}{8}} u \frac{1}{r^{2}}\right\|_{L_{x}^{2}}\left\|A_{\theta}\right\|_{L_{x}^{\infty}} \lesssim\left\|\varphi_{>\frac{1}{8}} u\right\|_{L_{x}^{2}}\|u\|_{L_{x}^{2}} \lesssim u 1, \\
& \left\|\varphi_{>\frac{1}{4}} \partial_{r}\left(N_{2}(u)\right)\right\|_{L_{x}^{2}} \lesssim\left\|\varphi_{>\frac{1}{4}} \frac{|u|^{2} u}{r^{2}}\right\|_{L_{x}^{2}}+\left\|\varphi_{>\frac{1}{4}} \frac{A_{\theta} \partial_{r} u}{r^{2}}\right\|_{L_{x}^{2}}+\left\|\varphi_{>\frac{1}{4}} \frac{A_{\theta} u}{r^{3}}\right\|_{L_{x}^{2}} \\
& \leq\left\|\varphi_{>\frac{1}{4}} N_{1}(u)\right\|_{L_{x}^{2}}+\left\|A_{\theta}\right\|_{L_{x}^{\infty}}\left\|\varphi_{>\frac{1}{8}} \partial_{r} u\right\|_{L_{x}^{2}} \lesssim_{u} 1 .
\end{aligned}
$$

Next, for (4.43) and (4.44), we need to be more careful so as to gain enough spatial decay. So here we use (4.60) instead of (4.61). Firstly, for $N_{1}(u)$,

$$
\begin{aligned}
\left\|\varphi_{>T} N_{1}(u)\right\|_{L_{x}^{1}} & \leq\left\|\varphi_{>\frac{T}{2}} u\right\|_{L_{x}^{2}}^{2}\left\|\varphi_{>\frac{T}{2}} r^{\frac{1}{2}} u\right\|_{L_{x}^{\infty} \| \varphi_{>\frac{T}{2}}} r^{-\frac{1}{2}} \|_{L_{x}^{\infty} \lesssim_{u} T^{-\frac{1}{2}}} \\
\left\|\varphi_{>T} \partial_{r}\left(N_{1}(u)\right)\right\|_{L_{x}^{1}} & \leq\left\|\varphi_{>\frac{T}{2}} \partial_{r} u\right\|_{L_{x}^{2}}\left\|\varphi_{>\frac{T}{2}} u\right\|_{L_{x}^{2}}\left\|\varphi_{>\frac{T}{2}} r^{\frac{1}{2}} u\right\|_{L_{x}^{\infty} \| \varphi_{>\frac{T}{2}}} r^{-\frac{1}{2}} \|_{L_{x}^{\infty}} \\
& \lesssim_{u} T^{-\frac{1}{2}} .
\end{aligned}
$$

For other nonlocal nonlinearities, we make use of their $r^{-2}$ spatial decay (see (4.64), (4.65) for decay of $A_{0}$ ). We merely show estimates for $N_{2}(u)$ and the others come in a similar way:

$$
\begin{aligned}
\left\|\varphi_{>T} N_{2}(u)\right\|_{L_{x}^{1}} & \lesssim\left\|A_{\theta}\right\|_{L_{x}^{\infty}}^{2}\left\|\varphi_{>\frac{T}{2}} u\right\|_{L_{x}^{2}}\left\|\varphi_{>} \frac{T}{2} r^{-2}\right\|_{L_{x}^{2}} \lesssim u T^{-1}, \\
\left\|\varphi_{>T} \partial_{r}\left(N_{2}(u)\right)\right\|_{L_{x}^{1}} & \lesssim\left\|\varphi_{>T}\left(\frac{N_{1}(u)}{r^{2}}+\frac{A_{\theta} \partial_{r} u}{r^{2}}+\frac{N_{2}(u)}{r}\right)\right\|_{L_{x}^{1}} \\
& \lesssim u T^{-\frac{5}{2}}+T^{-1}+T^{-2} \lesssim T^{-1} .
\end{aligned}
$$

This completes the proof of Proposition 4.10, and thus Theorem 1.6 with the arguments in Section 4.2.

\section{A. Elliptic theory for CSS}

\section{A.1. Self-dual case}

Proof of Proposition 2.1. The positivity of energy follows easily from

$$
E\left[\phi_{0}\right]=\frac{1}{2} \int\left|\boldsymbol{D}_{+} \phi_{0}\right|^{2} \geq 0 .
$$


For the rigidity of the null energy solution, note that $E\left[\phi_{0}\right]=0$ implies $D_{+} \phi_{0}=0$, i.e.

$$
\partial_{r} \phi_{0}-\frac{m+A_{\theta}\left[\phi_{0}\right]}{r} \phi_{0}=0 \quad \forall r>0 .
$$

Since $A_{\theta}[u]$ is real, this implies its radial part $u$ satisfies

$$
\partial_{r}|u|^{2}-\frac{2 m+2 A_{\theta}[u]}{r}|u|^{2}=0 .
$$

We claim that $u$ is not being a nonzero function implies $|u|>0$ for all $r>0$. Otherwise, there exists $r_{0} \in(0, \infty)$ such that $u\left(r_{0}\right)=0$, and then Gronwall's inequality shows

$$
|u|^{2}(r) \leq \exp \left(\int_{r_{0}}^{r} \frac{2 m+2 A_{\theta}[u]\left(r^{\prime}\right)}{r^{\prime}} d r^{\prime}\right)|u|^{2}\left(r_{0}\right)=0 \quad \forall r>0,
$$

and hence $|u| \equiv 0$, which is a contradiction.

Then, by representing the value of $u$ in polar coordinates, we see that the phase part is always constant, i.e. $u(r)=\rho(r) e^{i \gamma_{0}}$ for $\rho$ some real-valued function, and without loss of generality, we can assume $\rho>0$.

Next, by a change of coordinates, $v:=r^{-2 m} \rho^{2}, w:=\log v$. From (A.3), we see $w$ satisfies the following second-order ODE on $[0,+\infty)$ with one boundary condition:

$$
\begin{aligned}
w^{\prime \prime}+\frac{1}{r} w^{\prime}+r^{2 m} e^{w} & =0, \\
w^{\prime}(0) & =0 .
\end{aligned}
$$

Each initial value $w(0) \in \mathbb{R}$ uniquely determines a solution, the corresponding $\rho$ of which is the scaling of the soliton $Q$. Combined with local well-posedness theory, we complete the proof.

\section{A.2. Non-self-dual case}

Recall that when $g>1$, the energy here is

$$
E\left[\phi_{0}\right]=\frac{1}{2}\left\|\boldsymbol{D}_{x} \phi_{0}\right\|_{L^{2}}^{2}-\frac{g}{4}\left\|\phi_{0}\right\|_{L^{4}}^{4}=\frac{1}{2}\left\|\boldsymbol{D}_{+} \phi_{0}\right\|_{L^{2}}^{2}-\frac{g-1}{4}\left\|\phi_{0}\right\|_{L^{4}}^{4} .
$$

The threshold charge is defined as the minimization of the $L^{2}$ norm of an $m$-equivariant function with nonpositive energy.

Lemma A.1 ([44, Lemmas 7.2, 7.5]). Define

$$
c_{m, g}=\inf \left\{\|\phi\|_{L^{2}}: \phi \in H_{m}^{1} \backslash\{0\}, E[\phi] \leq 0\right\} .
$$

Then $c_{m, g}>0$, and

$$
c_{m, g}=\inf \left\{\|\phi\|_{L^{2}}: \phi \in H_{m}^{1} \backslash\{0\}, E[\phi]=0\right\} .
$$

The minimizer of (A.6) is a standing wave solution to (eCSS). 
Lemma A.2 ([44, Lemma 7.7]). Let $\phi \in H_{m}^{1} \backslash\{0\}$ with $E[\phi]=0,\|\phi\|_{L^{2}}=c_{m, g}$. Then there exists $\alpha \in \mathbb{R}$ such that $\psi(t, x):=e^{i \alpha t} \phi(x)$ is a solution of (eCSS).

Remark A.3. This result comes from the minimization problem of charge with restraint $E[\phi]=0$. If $E^{\prime}[\phi]=0$, we get the case $\alpha=0$, and otherwise the minimizer satisfies the Euler-Lagrange equation with $\alpha \neq 0$. We can further exclude the case $\alpha<0$ by [5, Proposition 4.2] and [6, Proposition 3.3].

Next we exclude the charge minimizer with negative energy.

Lemma A.4. If $\phi \in H_{m}^{1}$ with $E[\phi]<0$, then $\|\phi\|_{L^{2}}>c_{m, g}$.

Proof. From (A.5) we know $\|\phi\|_{L^{2}} \geq c_{m, g}$. So, by contradiction, suppose there exists $\phi \in H_{m}^{1}$ such that $E[\phi]<0$ and $\|\phi\|_{L^{2}}=c_{m, g}$. Note that for $\beta \in \mathbb{R}$,

$$
E[\beta \phi]=\frac{\beta^{2}}{2} \int_{\mathbb{R}^{2}}\left[\left|\partial_{r} \phi\right|^{2}+\frac{1}{r^{2}}\left(m^{2}-\beta^{2} \frac{1}{2} \int_{0}^{r}|\phi|^{2} s d s\right)^{2}|\phi|^{2}-\beta^{2} \frac{g}{2}|\phi|^{4}\right] d x
$$

continuously depends on $\beta$, so we can choose $\beta<1$ very close to 1 , such that $E[\beta \phi]<0$. Then

$$
\|\beta \phi\|_{L^{2}}=\beta c_{m, g}<c_{m, g},
$$

which contradicts (A.5).

Combining these two lemmas, we get the variational characterization of the nullenergy critical-charge solution in the non-self-dual case.

Proof of Proposition 2.2. The positivity of energy comes directly from Lemma A.4. For the rigidity, only note that if $E\left[\phi_{0}\right]=0$ and $\phi_{0}$ is nonzero, by Lemma A.1 we also have $\left\|\phi_{0}\right\|_{L^{2}} \geq c_{m, g}$, while the condition says $\left\|\phi_{0}\right\|_{L^{2}} \leq c_{m, g}$. So the equivalence holds, and Lemma A. 2 ensures that $\phi_{0}$ will generate a stationary wave or static solution.

Finally, we prove the exponential decay of any stationary wave satisfying (1.15) with $\alpha>0$. Take

$$
c_{u}(x):=\alpha+\frac{m^{2}}{r^{2}}+\frac{2 m}{r^{2}} A_{\theta}[u]+A_{0}[u]+\frac{1}{r^{2}} A_{\theta}[u]^{2}-g|u|^{2} .
$$

From the Strauss estimate $|u(r)| \lesssim r^{-1}$ and $A_{0}[u](r) \rightarrow 0$ as $r \rightarrow \infty$, we have $\mathscr{L} u:=$ $\left(\Delta-c_{u}(x)\right) u=0$ and $c_{u}(x) \in\left(\frac{\alpha}{2}, \frac{3 \alpha}{2}\right)$ on $B_{R}^{c}$ with $R$ large enough. So we can construct $v:=e^{-a|x|}$ with $a$ small enough such that $\mathscr{L} v \leq\left(\Delta-\frac{\alpha}{2}\right) v \leq 0$. Now we take $C$ large enough such that $u-v<0$ on $\partial B_{R}$, and the comparison theorem implies that $u \leq C v=$ $C e^{-a|x|}$.

\section{B. Covariant $H_{m}^{1}$ norm and equivariant Sobolev space}

We denote $B_{r}:=\left\{x \in \mathbb{R}^{2}:|x|<r\right\}$ and $B_{r}^{c}=\mathbb{R}^{2} \backslash B_{r}$ throughout this section. 
Proof of Lemma 3.1. Note that $A_{\theta}[f](r)=-\frac{1}{4 \pi}\|f\|_{L^{2}\left(B_{r}\right)}^{2}$ is decreasing. If $\|f\|_{L^{2}}^{2} \leq$ $2 \pi m$, then $A_{\theta}[f] \geq-\frac{m}{2}$ and obviously we have (3.2). Otherwise, take

$$
R:=\sup _{r \geq 0}\left\{A_{\theta}[f](r) \geq-\frac{m}{2}\right\} \in(0, \infty) .
$$

Then we have

$$
\|f\|_{L^{2}\left(B_{R}\right)}^{2}=2 \pi m
$$

And

$$
\begin{aligned}
\left\|\frac{1}{r}\left(m+A_{\theta}[f]\right) f\right\|_{L^{2}\left(B_{R}\right)}^{2} & \geq \frac{m^{2}}{4}\left\|\frac{1}{r} f\right\|_{L^{2}\left(B_{R}\right)}^{2}, \\
\left\|\frac{1}{r}\left(m+A_{\theta}[f]\right) f\right\|_{L^{2}\left(B_{R}\right)}^{2} & \geq \frac{1}{R^{2}}\|f\|_{L^{2}\left(B_{R}\right)}^{2} \frac{m^{2}}{4}=\frac{\pi m^{3}}{2 R^{2}}, \\
\left\|\frac{1}{r} f\right\|_{L^{2}\left(B_{R}^{c}\right)}^{2} & \leq \frac{1}{R^{2}}\|f\|_{L^{2}}^{2} .
\end{aligned}
$$

Inequalities (B.2) and (B.3) imply that

$$
\left\|\frac{1}{r} f\right\|_{L^{2}\left(B_{R}^{c}\right)}^{2} \leq \frac{2\|f\|_{L^{2}}^{2}}{\pi m^{3}}\left\|\frac{1}{r}\left(m+A_{\theta}[f]\right) f\right\|_{L^{2}\left(B_{R}\right)}^{2} .
$$

Estimate (3.2) for $\|f\|_{L^{2}}^{2} \geq 2 \pi m$ is established by combining (B.1) and (B.4).

For (3.3), we use the Strauss estimate (4.28) to estimate $A_{\theta}$ : for $f \in H_{m}^{1}$,

$$
\left|A_{\theta}[f](r)\right| \leq \frac{1}{2} \int_{0}^{r}\left\|f r^{\frac{1}{2}}\right\|_{L^{\infty}}^{2} d s \lesssim\left\|\partial_{r} f\right\|_{L^{2}}\|f\|_{L^{2}} r .
$$

Thus

$$
\left\|\frac{A_{\theta}[f]}{r} f\right\|_{L^{2}} \leq\left\|\frac{A_{\theta}[f]}{r}\right\|_{L^{\infty}}\|f\|_{L^{2}} \lesssim\|f\|_{L^{2}}^{2}\left\|\partial_{r} f\right\|_{L^{2}} .
$$

Combining (3.2) and (3.3), we get (3.4). For $m>0$,

$$
\begin{aligned}
\|f\|_{\dot{H}_{m}^{1}}^{2} & =\left\|\partial_{r} f\right\|_{L^{2}}^{2}+\left\|\frac{m}{r} f\right\|_{L^{2}}^{2} \\
& \lesssim_{m,\|f\|_{L^{2}}}\left\|\partial_{r} f\right\|_{L^{2}}^{2}+\left\|\frac{m+A_{\theta}[f]}{r} f\right\|_{L^{2}}^{2}=\left\|\boldsymbol{D}_{x} f\right\|_{L^{2}}^{2}, \\
\left\|\boldsymbol{D}_{x} f\right\|_{L^{2}}^{2} & =\left\|\partial_{r} f\right\|_{L^{2}}^{2}+\left\|\frac{m+A_{\theta}[f]}{r} f\right\|_{L^{2}}^{2} \\
& \leq\left\|\partial_{r} f\right\|_{L^{2}}^{2}+\left(1+\frac{1}{4 \pi m}\|f\|_{L^{2}}^{2}\right)\left\|\frac{m}{r} f\right\|_{L^{2}}^{2} \lesssim\|f\|_{\dot{H}_{m}^{1}}^{2} .
\end{aligned}
$$

For $m=0$, the "less than" direction is immediate, while the other comes from (3.3).

Proof of Lemma 3.2. Firstly, since

$$
\partial_{r}=\frac{x_{1}}{|x|} \partial_{1}+\frac{x_{2}}{|x|} \partial_{2},
$$


and $\frac{x_{1}}{|x|}, \frac{x_{2}}{|x|}$ are $L^{\infty}$ functions, we immediately have (3.5) from the weak convergence of $\partial_{1} f_{n}$ and $\partial_{2} f_{n}$.

Next we show (3.7) for $m \geq 1$.

Let $\left\|f_{n}\right\|_{H^{1}},\|f\|_{H^{1}} \leq M$. Note that $f_{n} \rightarrow f$ in $L^{2}$, and that $\frac{1}{r}$ is bounded away from the origin. We have, for all $R>0$,

$$
\frac{1}{r} f_{n} \rightarrow \frac{1}{r} f \quad \text { in } L^{2}\left(B_{R}^{c}\right)
$$

Now we can show that for any $g \in L^{2}$,

$$
\left(\frac{1}{r} f_{n}, g\right)_{L^{2}} \rightarrow\left(\frac{1}{r} f, g\right)_{L^{2}} \text { as } n \rightarrow \infty
$$

Notice that $\frac{1}{r} f_{n}, \frac{1}{r} f$ are uniformly bounded in $L^{2}$ by $M$ (for $m \geq 1$ ). We then have, for all $R>0$, from (B.6),

$$
\begin{aligned}
\left|\left(\frac{1}{r}\left(f_{n}-f\right), g\right)_{L^{2}}\right| & \leq\left|\left(\frac{1}{r}\left(f_{n}-f\right), g\right)_{L^{2}\left(B_{R}^{c}\right)}\right|+\left|\left(\frac{1}{r}\left(f_{n}-f\right), g\right)_{L^{2}\left(B_{R}\right)}\right| \\
& \leq o_{n}(1)+2 M\|g\|_{L^{2}\left(B_{R}\right)} .
\end{aligned}
$$

From the arbitrariness of $R>0$, (B.7) is confirmed. That is the weak convergence in (3.7).

Finally, we prove (3.6) for $m \geq 0$. Equation (3.8) directly follows from (3.5)-(3.7).

Noting that $\frac{A_{\theta}\left[f_{n}\right]}{r}, \frac{A_{\theta}[f]}{r}$ are uniformly $L^{\infty}$ bounded away from the origin, using the same strategy as above, we only need to prove the following two things:

(1) $\frac{A_{\theta}\left[f_{n}\right]}{r} f_{n}, \frac{A_{\theta}[f]}{r} f$ are uniformly bounded in $L^{2}$.

(2) For all $R>0$,

$$
\frac{A_{\theta}\left[f_{n}\right]}{r} f_{n} \rightarrow \frac{A_{\theta}\left[f_{n}\right]}{r} f \quad \text { in } B_{R}^{c}
$$

Item (1) follows (3.3) in Lemma 3.1, noticing that $f_{n}, f$ are uniformly bounded in $H^{1}$.

For (2), we again take a $g \in L^{2}$ and try to derive the convergence of the inner product. From the $m$-equivariance assumption, without loss of generosity, we may take $g \in L_{\text {rad }}^{2}$. Moreover, we can take $g \in C_{c \text {,rad }}^{\infty}$ as a test function by the density argument:

$$
\begin{aligned}
\left(\frac{1}{r} A_{\theta}\left[f_{n}\right] f_{n}, g\right)_{L^{2}\left(B_{R}^{c}\right)} & =\int_{R}^{\infty} \frac{1}{r} f_{n}(r) \bar{g}(r) \int_{0}^{r}\left|f_{n}\right|^{2}(s) s d s r d r \\
& =\int_{0}^{\infty}\left|f_{n}\right|^{2}(s) s\left(\int_{\max \{R, s\}}^{\infty} f_{n}(r) \bar{g}(r) d r\right) d s \\
& =\int_{0}^{\infty}\left|f_{n}\right|^{2}(s) s G_{n, R}(s) d s .
\end{aligned}
$$

Then from the weak convergence (B.6) for general $m \geq 0$, we have a pointwise convergence

$$
G_{n, R}(s)=\left(\frac{1}{r} f_{n}, g\right)_{L^{2}\left(B_{\max \{R, s\}}^{c}\right)} \rightarrow\left(\frac{1}{r} f, g\right)_{L^{2}\left(B_{\max \{R, s\}}^{c}\right)}=: G_{R}(s) \quad \forall s \geq 0 .
$$


So $G_{n, R}$ and $G_{R}(s)$ are uniformly bounded in $L^{\infty}$. And compact support of $g$ implies that $G_{n, R}, G_{R}$ are also compactly supported. So they are uniformly bounded in $L^{p}$ for any $p \in[1, \infty]$. By dominated convergence,

$$
G_{n, R} \rightarrow G_{R} \text { in } L^{2} .
$$

Also, from the compact embedding $H_{\text {rad }}^{1}\left(\mathbb{R}^{2}\right) \hookrightarrow L^{4}\left(\mathbb{R}^{2}\right)$, we have

$$
f_{n} \rightarrow f \quad \text { in } L^{4} \text {. }
$$

Now

$$
\begin{aligned}
& \left|\left(\frac{1}{r} A_{\theta}\left[f_{n}\right] f_{n}, g\right)_{L^{2}\left(B_{R}^{c}\right)}-\left(\frac{1}{r} A_{\theta}[f] f, g\right)_{L^{2}\left(B_{R}^{c}\right)}\right| \\
& \quad \leq\left|\left(G_{n, R},\left|f_{n}\right|^{2}\right)_{L^{2}}-\left(G_{R},|f|^{2}\right)_{L^{2}}\right| \\
& \quad \leq\left|\left(G_{n, R}-G_{R},\left|f_{n}\right|^{2}\right)_{L^{2}}\right|+\left|\left(G_{R} f_{n}, f_{n}-f\right)_{L^{2}}\right|+\left|\left(G_{R} \bar{f}, \overline{f_{n}-f}\right)_{L^{2}}\right| \\
& \quad \leq\left\|G_{n, R}-G_{R}\right\|_{L^{2}}\left\|f_{n}\right\|_{L^{4}}^{2}+\left\|G_{R}\right\|_{L^{2}}\left(\left\|f_{n}\right\|_{L^{4}}+\|f\|_{L^{4}}\right)\left\|f_{n}-f\right\|_{L^{4}} \\
& \quad \rightarrow 0 \quad \text { as } n \rightarrow \infty .
\end{aligned}
$$

That finishes the proof of (2) and concludes the proof of Lemma 3.2.

Proof of Lemma 3.3. Consider $\boldsymbol{D}_{+} v_{n}-\boldsymbol{D}_{+} v \rightarrow 0$ in $L^{2}$. Note that

$$
\begin{aligned}
\left\|\boldsymbol{D}_{+} v_{n}-\boldsymbol{D}_{+} v\right\|_{L^{2}}^{2} & =\left\|\partial_{r}\left(v_{n}-v\right)-\frac{m}{r}\left(v_{n}-v\right)-\left(\frac{A_{\theta}\left[v_{n}\right]}{r} v_{n}-\frac{A_{\theta}[v]}{r} v\right)\right\|_{L^{2}}^{2} \\
& \gtrsim\left\|\partial_{r}\left(v_{n}-v\right)-\frac{m+A_{\theta}[v]}{r}\left(v_{n}-v\right)\right\|_{L^{2}}^{2}-\left\|\frac{A_{\theta}\left[v_{n}\right]-A_{\theta}[v]}{r} v_{n}\right\|_{L^{2}}^{2} \\
& :=\mathrm{I}_{n}-\mathrm{II}_{n} .
\end{aligned}
$$

Using integration by parts,

$$
\begin{aligned}
\mathrm{I}_{n}= & \int_{0}^{\infty}\left[\left|\partial_{r}\left(v_{n}-v\right)\right|^{2}+\left|\frac{m+A_{\theta}[v]}{r}\left(v_{n}-v\right)\right|^{2}\right. \\
& \left.\quad-2 \operatorname{Re}\left(\overline{\partial_{r}\left(v_{n}-v\right)}\left(v_{n}-v\right) \frac{m+A_{\theta}[v]}{r}\right)\right] r d r \\
= & \left\|\partial_{r}\left(v_{n}-v\right)\right\|_{L^{2}}^{2}+\left\|\frac{m+A_{\theta}[v]}{r}\left(v_{n}-v\right)\right\|_{L^{2}}^{2}-\frac{1}{2} \int_{0}^{\infty}\left|v_{n}-v\right|^{2}|v|^{2} r d r \\
:= & \mathrm{I}_{n}^{1}+\mathrm{I}_{n}^{2}-\frac{1}{2} \mathrm{I}_{n}^{3} .
\end{aligned}
$$

From convergence in $L^{2}$ and $L^{4}$, and the uniform bound in $H^{1}$ for $v_{n}$ and $v$, we know

$$
\begin{aligned}
\mathrm{II}_{n} & \leq\left\|A_{\theta}\left[v_{n}\right]-A_{\theta}[v]\right\|_{L^{\infty}}^{2}\left\|\frac{v_{n}}{r}\right\|_{L^{2}}^{2} \\
& \lesssim\left\|v_{n}-v\right\|_{L^{2}}^{2}\left\|v_{n}+v\right\|_{L^{2}}^{2}\left\|\frac{v_{n}}{r}\right\|_{L^{2}}^{2} \rightarrow 0, \\
\mathrm{I}_{n}^{3} & \lesssim\left\|v_{n}-v\right\|_{L^{4}}^{2}\|v\|_{L^{4}}^{2} \rightarrow 0 .
\end{aligned}
$$


Thus

$$
\mathrm{I}_{n}^{1}+\mathrm{I}_{n}^{2} \rightarrow 0
$$

which implies that

$$
\partial_{r} v_{n} \rightarrow \partial_{r} v \quad \text { in } L^{2}
$$

And for $m \geq 1$, taking $r_{0}>0$ to be

$$
r_{0}:=\sup _{r \geq 0}\left\{A_{\theta}[v](r) \geq-\frac{m}{2}\right\}>0,
$$

we have

$$
\frac{1}{r} v_{n} \rightarrow \frac{1}{r} v \quad \text { in } L^{2}\left(\left\{|x| \leq r_{0}\right\}\right)
$$

Combined with $v_{n} \rightarrow v$ in $L^{2}$, we know for $m \geq 1$,

$$
\frac{1}{r} v_{n} \rightarrow \frac{1}{r} v \text { in } L^{2}
$$

Convergences (B.9) and (B.10) imply the $\dot{H}^{1}$ convergence of $v_{n}$ to $v$.

Acknowledgments. We are grateful to Kihyun Kim and Soonsik Kwon for helpful discussions and remarks.

Funding. The authors are supported by the NSF of China (nos. 12071010, 11631002).

\section{References}

[1] V. Banica, Remarks on the blow-up for the Schrödinger equation with critical mass on a plane domain. Ann. Sc. Norm. Super. Pisa Cl. Sci. (5) 3 (2004), no. 1, 139-170 Zbl 1170.35528 MR 2064970

[2] I. Bejenaru, A. Ionescu, C. Kenig, and D. Tataru, Equivariant Schrödinger maps in two spatial dimensions. Duke Math. J. 162 (2013), no. 11, 1967-2025 Zbl 1326.35087 MR 3090782

[3] H. Berestycki and P.-L. Lions, Existence d'ondes solitaires dans des problèmes nonlinéaires du type Klein-Gordon. C. R. Acad. Sci. Paris Sér. A-B 288 (1979), no. 7, A395-A398 Zbl 0397.35024 MR 552061

[4] L. Bergé, A. De Bouard, and J.-C. Saut, Blowing up time-dependent solutions of the planar, Chern-Simons gauged nonlinear Schrödinger equation. Nonlinearity 8 (1995), no. 2, 235-253 Zbl 0822.35125 MR 1328596

[5] J. Byeon, H. Huh, and J. Seok, Standing waves of nonlinear Schrödinger equations with the gauge field. J. Funct. Anal. 263 (2012), no. 6, 1575-1608 Zbl 1248.35193 MR 2948224

[6] J. Byeon, H. Huh, and J. Seok, On standing waves with a vortex point of order $N$ for the nonlinear Chern-Simons-Schrödinger equations. J. Differential Equations 261 (2016), no. 2, 1285-1316 Zbl 1342.35321 MR 3494398

[7] T. Cazenave, Semilinear Schrödinger equations. Courant Lect. Notes Math. 10, New York University, Courant Institute of Mathematical Sciences, New York; American Mathematical Society, Providence, RI, 2003 Zbl 1055.35003 MR 2002047 
[8] N.-H. Chang, J. Shatah, and K. Uhlenbeck, Schrödinger maps. Comm. Pure Appl. Math. 53 (2000), no. 5, 590-602 Zbl 1028.35134 MR 1737504

[9] R. M. Chen and D. Spirn, Symmetric Chern-Simons-Higgs vortices. Comm. Math. Phys. 285 (2009), no. 3, 1005-1031 Zbl 1171.81020 MR 2470914

[10] R. Côte, C. E. Kenig, A. Lawrie, and W. Schlag, Characterization of large energy solutions of the equivariant wave map problem: I. Amer. J. Math. 137 (2015), no. 1, 139-207 Zbl 1315.35130 MR 3318089

[11] H. J. de Vega and F. A. Schaposnik, Electrically charged vortices in nonabelian gauge theories with Chern-Simons term. Phys. Rev. Lett. 56 (1986), no. 24, 2564-2566 MR 845964

[12] S. Deser, R. Jackiw, and S. Templeton, Topologically massive gauge theories. Ann. Physics 140 (1982), no. 2, 372-411 MR 665601

[13] B. Dodson, Global well-posedness and scattering for the mass critical nonlinear Schrödinger equation with mass below the mass of the ground state. Adv. Math. 285 (2015), 1589-1618 Zbl 1331.35316 MR 3406535

[14] G. Dunne, Self-dual Chern-Simons theories. Lect. Notes Phys. 36, Springer, Berlin, 2009 Zbl 0834.58001

[15] T. Duyckaerts and F. Merle, Dynamics of threshold solutions for energy-critical wave equation. Int. Math. Res. Pap. IMRP (2008), Art ID rpn002 Zbl 1159.35043 MR 2470571

[16] T. Duyckaerts and F. Merle, Dynamic of threshold solutions for energy-critical NLS. Geom. Funct. Anal. 18 (2009), no. 6, 1787-1840 Zbl 1232.35150 MR 2491692

[17] T. Duyckaerts and S. Roudenko, Threshold solutions for the focusing 3D cubic Schrödinger equation. Rev. Mat. Iberoam. 26 (2010), no. 1, 1-56 Zbl 1195.35276 MR 2662148

[18] Z. F. Ezawa, M. Hotta, and A. Iwazaki, Breathing vortex solitons in nonrelativistic ChernSimons gauge theory. Phys. Rev. Lett. 67 (1991), no. 4, 411-414 Zbl 0990.81560 MR 1114940

[19] Z. F. Ezawa, M. Hotta, and A. Iwazaki, Nonrelativistic Chern-Simons vortex solitons in external magnetic field. Phys. Rev. D (3) 44 (1991), no. 2, 452-463

[20] I. S. Gradshteyn and I. M. Ryzhik, Table of integrals, series, and products. Academic Press, Amsterdam, 2015 Zbl 1300.65001 MR 3307944

[21] T. Hmidi and S. Keraani, Blowup theory for the critical nonlinear Schrödinger equations revisited. Int. Math. Res. Not. (2005), no. 46, 2815-2828 Zbl 1126.35067 MR 2180464

[22] H. Huh, Blow-up solutions of the Chern-Simons-Schrödinger equations. Nonlinearity 22 (2009), no. 5, 967-974 Zbl 1173.35313 MR 2501032

[23] H. Huh, Energy solution to the Chern-Simons-Schrödinger equations. Abstr. Appl. Anal. (2013), Art. ID 590653 Zbl 1276.35138 MR 3035224

[24] H. Huh and J. Seok, The equivalence of the Chern-Simons-Schrödinger equations and its selfdual system. J. Math. Phys. 54 (2013), no. 2, Art. ID 021502 Zbl 1280.81042 MR 3076362

[25] S. Ibrahim, N. Masmoudi, and K. Nakanishi, Threshold solutions in the case of mass-shift for the critical Klein-Gordon equation. Trans. Amer. Math. Soc. 366 (2014), no. 11, 5653-5669 Zbl 1302.35260 MR 3256178

[26] R. Jackiw and S.-Y. Pi, Time-dependent Chern-Simons solitons and their quantization. Phys. Rev. D (3) 44 (1991), no. 8, 2524-2532 MR 1132645

[27] R. Jackiw, S.-Y. Pi, and E. J. Weinberg, Topological and nontopological solitons in relativistic and nonrelativistic Chern-Simons theory. In Particles, strings and cosmology (Boston, MA, 1990), pp. 573-588, World Scientific, River Edge, NJ, 1991 MR 1170608

[28] R. Jackiw and S. Templeton, How super-renormalizable interactions cure their infrared divergences. Phys. Rev. D 23 (1981), no. 10, 2291-2304 
[29] R. Jackiw and E. J. Weinberg, Self-dual Chern-Simons vortices. Phys. Rev. Lett. 64 (1990), no. 19, 2234-2237 Zbl 1050.81595 MR 1050530

[30] J. Jendrej and A. Lawrie, Two-bubble dynamics for threshold solutions to the wave maps equation. Invent. Math. 213 (2018), no. 3, 1249-1325 Zbl 1411.35205 MR 3842064

[31] R. Killip, D. Li, M. Visan, and X. Zhang, Characterization of minimal-mass blowup solutions to the focusing mass-critical NLS. SIAM J. Math. Anal. 41 (2009), no. 1, 219-236 Zbl 1184.35293 MR 2505858

[32] R. Killip, T. Tao, and M. Visan, The cubic nonlinear Schrödinger equation in two dimensions with radial data. J. Eur. Math. Soc. (JEMS) 11 (2009), no. 6, 1203-1258 Zbl 1187.35237 MR 2557134

[33] R. Killip, M. Visan, and X. Zhang, The mass-critical nonlinear Schrödinger equation with radial data in dimensions three and higher. Anal. PDE 1 (2008), no. 2, 229-266

Zbl 1171.35111 MR 2472890

[34] K. Kim and S. Kwon, Construction of blow-up manifolds to the equivariant self-dual ChernSimons-Schrödinger equation. 2020, arXiv:2009.02943

[35] K. Kim and S. Kwon, On pseudoconformal blow-up solutions to the self-dual Chern-SimonsSchrödinger equation: existence, uniqueness, and instability. Mem. Amer. Math. Soc., to appear

[36] K. Kim, S. Kwon, and S.-J. Oh, Blow-up dynamics for smooth finite energy radial data solutions to the self-dual Chern-Simons-Schrödinger equation. 2020, arXiv:2010.03252

[37] M. K. Kwong, Uniqueness of positive solutions of $\Delta u-u+u^{p}=0$ in $\mathbf{R}^{n}$. Arch. Rational Mech. Anal. 105 (1989), no. 3, 243-266 Zbl 0676.35032 MR 969899

[38] D. Li and X. Zhang, Dynamics for the energy critical nonlinear Schrödinger equation in high dimensions. J. Funct. Anal. 256 (2009), no. 6, 1928-1961 Zbl 1162.35070 MR 2498565

[39] D. Li and X. Zhang, On the focusing mass critical problem in six dimensions with splitting spherically symmetric initial data. Dyn. Partial Differ. Equ. 7 (2010), no. 4, 345-373 Zbl 1220.35162 MR 2780249

[40] D. Li and X. Zhang, Regularity of almost periodic modulo scaling solutions for mass-critical NLS and applications. Anal. PDE 3 (2010), no. 2, 175-195 Zbl 1225.35220 MR 2657453

[41] D. Li and X. Zhang, Dynamics for the energy critical nonlinear wave equation in high dimensions. Trans. Amer. Math. Soc. 363 (2011), no. 3, 1137-1160 Zbl 1221.35248 MR 2737260

[42] D. Li and X. Zhang, On the rigidity of solitary waves for the focusing mass-critical NLS in dimensions $d \geq 2$. Sci. China Math. 55 (2012), no. 2, 385-434 Zbl 1238.35146 MR 2886544

[43] Z. M. Lim, Large data well-posedness in the energy space of the Chern-Simons-Schrödinger system. J. Differential Equations 264 (2018), no. 4, 2553-2597 Zbl 1386.35381 MR 3737847

[44] B. Liu and P. Smith, Global wellposedness of the equivariant Chern-Simons-Schrödinger equation. Rev. Mat. Iberoam. 32 (2016), no. 3, 751-794 Zbl 1352.35161 MR 3556051

[45] B. Liu, P. Smith, and D. Tataru, Local wellposedness of Chern-Simons-Schrödinger. Int. Math. Res. Not. IMRN (2014), no. 23, 6341-6398 Zbl 1304.35649 MR 3286341

[46] L. Martina, O. K. Pashaev, and G. Soliani, Self-dual Chern-Simons solitons in nonlinear $\sigma$ models. Modern Phys. Lett. A 8 (1993), no. 34, 3241-3250 Zbl 1021.81661 MR 1249658

[47] F. Merle, On uniqueness and continuation properties after blow-up time of self-similar solutions of nonlinear Schrödinger equation with critical exponent and critical mass. Comm. Pure Appl. Math. 45 (1992), no. 2, 203-254 Zbl 0767.35084 MR 1139066 
[48] F. Merle, Determination of blow-up solutions with minimal mass for nonlinear Schrödinger equations with critical power. Duke Math. J. 69 (1993), no. 2, 427-454 Zbl 0808.35141 MR 1203233

[49] C. Miao, Y. Wu, and G. Xu, Dynamics for the focusing, energy-critical nonlinear Hartree equation. Forum Math. 27 (2015), no. 1, 373-447 Zbl 1332.35227 MR 3334067

[50] S.-J. Oh and F. Pusateri, Decay and scattering for the Chern-Simons-Schrödinger equations. Int. Math. Res. Not. IMRN (2015), no. 24, 13122-13147 Zbl 1332.35341 MR 3436140

[51] S. K. Paul and A. Khare, Charged vortices in an abelian Higgs model with Chern-Simons term. Phys. Lett. B 174 (1986), no. 4, 420-422 MR 855612

[52] M. Struwe, Equivariant wave maps in two space dimensions. Comm. Pure Appl. Math. 56 (2003), no. 7, 815--823 Zbl 1033.53019 MR 1990477

[53] T. Tao, M. Visan, and X. Zhang, Minimal-mass blowup solutions of the mass-critical NLS. Forum Math. 20 (2008), no. 5, 881-919 Zbl 1154.35085 MR 2445122

[54] M. I. Weinstein, Nonlinear Schrödinger equations and sharp interpolation estimates. Comm. Math. Phys. 87 (1982/83), no. 4, 567-576 Zbl 0527.35023 MR 691044

[55] M. I. Weinstein, On the structure and formation of singularities in solutions to nonlinear dispersive evolution equations. Comm. Partial Differential Equations 11 (1986), no. 5, 545-565 Zbl 0596.35022 MR 829596

[56] F. Wilczek, Fractional statistics and anyon superconductivity. World Scientific, Teaneck, NJ, 1990 Zbl 0709.62735 MR 1081990

Received 7 December 2020; revised 18 June 2021; accepted 21 July 2021.

\section{Zexing Li}

School of Mathematical Sciences, Peking University, Beijing 100871, China;

lizexing@pku.edu.cn

\section{Baoping Liu}

Beijing International Center for Mathematical Research, Peking University, Beijing 100871,

China; baoping@math.pku.edu.cn 